\title{
Acknowledgment to Reviewers of Mathematics in 2021
}

\section{Mathematics Editorial Office}

MDPI AG, St. Alban-Anlage 66, 4052 Basel, Switzerland

Rigorous peer-reviews are the basis of high-quality academic publishing. Thanks to the great efforts of our reviewers, Mathematics was able to maintain its standards for the high quality of its published papers. Thanks to the contribution of our reviewers, in 2021, the median time to first decision was 19 days and the median time to publication was 38 days. The editors would like to extend their gratitude and recognition to the following reviewers for their precious time and dedication, regardless of whether the papers they reviewed were finally published:

A. A. El-Bary

A. A. El-Deeb

A. Alexandre Trindade

A. Di Bucchianico

A. Dilek Maden

A. Gowrisankar

A. H. Alamoodi

A. Krivoshein

A. M. Rashad

A. S. M. Monjurul Hasan

A. V. Plotnikov

Aakil Caunhye

Aamir Saghir

Aashwin Mishra

Abbas Abbaszadeh Shahri

Abbas Kareem Wanas

Abbas Mardani

Abbas Roozbahani

Abbas-Ali Zamani

Abdallah El-Hamidi

Abd-Allah Hyder

Abdel Azziz Mennouni

Abdel Moneim Lashin

Abdelalim Elsadany

Abdeldjalil Ouahabi

Abdelfattah Y. A. Soliman

Abdelhakim Chillali

Abdelhalim Ebaid

Abdeljalil Métioui

Abdellah Chehri

Abdelmejid Bayad

Abdelouaheb Ardjouni

Abderrahim Wakif

Abderrahmane Nitaj
Abdolhossein Sarafzadeh

Abdolrahman Razani

Abdolsaeed Toomaj

Abdon Atangana

Abdon Choque Rivero

Abdul Ali

Abdul Majeed

Abdul Samad Khan

Abdullah Akkurt

Abdullah Kargin

Abdullah Shaheen

Abdullahi Adem

Abdus Saboor

Abel Cabrera Martínez

Abhijit Datta Banik

Abhilash Somayajula

Abhrajit Sengupta

Abiodun Ismail Lawal

Abla Smahi

Abolfazl Shirazi

Abraham Arenas

Abror Khudoyberdiyev

Abu Sayed Chowdhury

Abul B. M. Baki

Achyutha Krishnamoorthy

Adam Ciszkiewicz

Adam Deptuła

Adam Długosz

Adam Gadomski

Adam Głowacz

Adam Jakubowski

Adam Karbowski

Adam Kiersztyn

Adam Kolkiewicz 
Adam Lipowski

Adam Marchewka

Adam Marszk

Adam Rosiński

Adam Seliga

Adam Śliwiński

Adam Sulich

Adam Szromek

Adara-Monica Blaga

Addi Ait-Mlouk

Addolorata Marasco

Adel Ouannas

Adela Mihai

Adeleke Timothy Ademola

Adeolu Taiwo

Adewuyi Ayodele Adeyinka

Adil Jhangeer

Adil Usman

Aditya Kumar Sahu

Adolfo Ballester-Bolinches

Adolfo Maza

Adrià Asensio

Adrian Amor-Martin

Adrian Burlacu

Adrian Cantemir Calin

Adrian Deaconu

Adrián García-Gutiérrez

Adrian Gligor

Adrian Holhos

Adrian Kapczyński

Adrian Pop

Adrián Ruiz

Adriana Ana Maria Davidescu

Adriana Brancaccio

Adriana Catas

Adriana Peña Pérez Negron

Adriana Tiron-Tudor

Adrianna Kozierkiewicz

Adriano Barra

Adriano Decarli

Adrijana Visnjic Jevtic

Afshin Akhshani

Agárdi Anita

Agha Kashif

Ágnes Vathy-Fogarassy

Agnieszka B. Malinowska

Agnieszka Barczak

Agnieszka Gozdek

Agnieszka Konys

Agnieszka Martyna

Agnieszka Wielgus
Agnieszka Wiszniewska-Matyszkiel

Agnieszka Wnuk

Agostino Cortesi

Agoston Restas

Agus Suryanto

Ahmad El-Kharouf

Ahmad ElMoslimany

Ahmad Golbabai

Ahmad Jahanbakhshi

Ahmad Samed Al-Adwan

Ahmad Taher Azar

Ahmad Zeeshan

Ahmed Abdelaziz

Ahmed Elsawah

Ahmed Gamal Ibrahim

Ahmed Mostafa

Ahmed Salem

Ahmed Shihab Albahri

Ahmed Zidna

Ahmet Cagdas Seckin

Ahmet Ocak Akdemir

Aidin Salamazadeh

Aihua Wood

Aileni Raluca Maria

Airlane Alencar

Aivars Aboltinš

Ajeet Ram Pathak

Akaki Mamageishvili

Akash Saxena

Akbar Ali

Akbar Zada

Åke Brännström

Akebo Yamakami

Akhil Kandhari

Akhmad Habibi

Akihide Saimoto

Akindele Adebayo Mebawondu

Akio Matsumoto

Akio Tsuneda

Akira Otsuki

Ákos Pintér

Akram Ali

Akshay Kumar

Al Jupri

Ala Amourah

Alagar Karthick

Alain Vande Wouwer

Alan Filipin

Alan Robins

Albert Cohen

Alberto Arteta 
Alberto Fernandez

Alberto Fiorenza

Alberto Gambaruto

Alberto Magrenan

Alberto Pedrouzo-Ulloa

Alberto Rodríguez-Martínez

Alberto Romero Ania

Alberto Simões

Albina Orlando

Albrecht Petzoldt

Aldo Corbellini

Aldo Garay

Alecksey Anuchin

Alef Sterk

Alejandro Bustos

Alejandro Cornejo

Alejandro Estrada-Moreno

Alejandro Rodríguez-Collado

Alejandro Rodríguez-Molina

Alejandro Santiago

Aleka Papadopoulou

Aleksandar Erceg

Aleksandar R. Miković

Aleksandar Shurbevski

Aleksander Aristovnik

Aleksander Jakimowicz

Aleksandr Cariow

Aleksandr I. Kozhanov

Aleksandr Kutukov

Aleksandr Leonidovich Kazakov

Aleksandr Mikhalev

Aleksandr Rakhmangulov

Aleksandr Tynda

Aleksandra Gawel

Aleksandra Kawala-Sterniuk

Aleksandra Łuczak

Aleksandra Tutueva

Aleksandra Wójcicka-Wójtowicz

Aleksandras Krylovas

Aleksei Kondratev

Aleksei Pavlovich Mashtakov

Aleksejus Mihalkovich

Aleksey F. Rogachev

Aleksey I. Shinkevich

Aleksey Telyakovskiy

Alena Hošpesová

Alessandro Bogliolo

Alessandro Cammarata

Alessandro Coretti

Alessandro Fasana

Alessandro Formisano
Alessandro Lazzeri

Alessandro Maria Selvitella

Alessandro Mengarelli

Alessandro Mingotti

Alessandro N. Vargas

Alessandro Niccolai

Alessandro Severino

Alessandro Sitta

Alessandro Stecco

Alessandro Stefano

Alessia Cogato

Alessio Corti

Alessio De Rango

Alex Karagrigoriou

Alex V. Zakharov

Alexander Aksenov

Alexander Aleksandrovich Safonov

Alexander Aptekarev

Alexander Becker

Alexander Bogdanov

Alexander Brauneis

Alexander Danilov

Alexander Dudin

Alexander Eliseev

Alexander Farrugia

Alexander Felshtyn

Alexander Grigoriev

Alexander H. Pesch

Alexander Harrison

Alexander Hošovský

Alexander Hvatov

Alexander Iomin

Alexander Kalashnikov

Alexander Lachmann

Alexander Maz Machado

Alexander McNeil

Alexander Moiseev

Alexander P. Lyapin

Alexander Pchelintsev

Alexander Pisarchik

Alexander Rauh

Alexander Robitzsch

Alexander Schaum

Alexander Shapovalov

Alexander Trounev

Alexander V. Lapin

Alexander V. Mantzaris

Alexander Zeifman

Alexander Matasov

Alexandra Galhano

Alexandra Gavina 
Alexandra Horobet

Alexandra Kashchenko

Alexandra Moshou

Alexandre B. Nassif

Alexandre G. De Brevern

Alexandre Morais Nunes

Alexandre Rodrigues

Alexandre Souto Martinez

Alexandre Talon

Alexandru Isaic-Maniu

Alexandru Onea

Alexandru-Codrin Ionescu

Alexei L'vovich Semenov

Alexei Sergeevich Shvedov

Alexey A. Melnikov

Alexey Beskopylny

Alexey E. Madison

Alexey Ivutin

Alexey Lobanov

Alexey Lukashov

Alexey Mikhaylov

Alexey S. Eremin

Alexey Shevyakov

Alexey V. Bosov

Alexey V. Shvetsov

Alexey Yu Bykovsky

Alexey Yu Popkov

Alexis Morris

Alfonso Gómez-Espinosa

Alfonso Infante

Alfredas Račkauskas

Alfredo Hernández

Alfredo Marvão Pereira

Alfredo Milani

Alfredo Peris

Alhaji Cherif

Ali Abdul-Aziz

Ali Ahmadian

Ali Akbari

Ali Akgul

Ali Amiryousefi

Ali Cemal Benim

Ali Deriszadeh

Ali Djerioui

Ali Ebadi Torkayesh

Ali Ebrahimnejad

Ali Elsherbeni Takieldeen

Ali Eltamaly

Ali Fareed Jameel

Ali Haji-Badali

Ali Johari
Ali Lalbakhsh

Ali Mohammadi

Ali Mostafaeipour

Ali Pourzangbar

Ali Raza

Ali Reza Yarahmadi Bafghi

Ali Riza Ekti

Ali Shahidinejad

Ali Shokri

Ali Yekkehkhany

Ali Yousef

Alice Barana

Alim Sukhtayev

Alin Suciu

Alina Barbulescu

Alina Cristiana Gavrilut

Alina Dobrogowska

Alina Ilinca Lazu

Alina Minea

Alina-Mihaela Patriciu

Aliona Dreglea

Alireza Khalili Golmankhaneh

Alireza Khastan

Alireza Ranjbar-Motlagh

Alireza Rezvanian

Alireza Sharifi

Alison J. McMillan

Aliya Işıksal

Aliyu Aliyu

Aljoša Peperko

Allal Guessab

Allen W. Herman

Alma Alanis

Almudena P. Márquez

Alon Davidy

Alper Aksac

Alptekin Ulutas

Alun Lloyd

Álvaro Aguilar González

Álvaro Antón

Álvaro Briz-Redón

Álvaro Gutiérrez

Álvaro Lozano Rojo

Alvaro Luiz Neuenfeldt Júnior

Alyona Zamyshlyaeva

Alzbeta Michalikova

Alzira Mota

Amalendu Ghosh

Aman Ullah

Amanda Obery

Amar Debbouche 
Amedeo Altavilla

Amelia Bădică

Amelia Carolina Sparavigna

Amelia Giuseppina Nobile

Americo Correia

Ameya Jagtap

Amilcar Meneses-Viveros

Amin Jajarmi

Amin Mohammadpour Shotorbani

Amine Rghioui

Amine Roukh

Aming Li

Amir Asif

Amir Benzaoui

Amir Ghasem Ghazanfari

Amir H. Gandomi

Amir Mahdiyar

Amir Mosavi

Amir Seyyedabbasi

Amir Teimourian

Amirreza Masoodi

Amit K. Verma

Amit Kumar

Amit Prakash

Amjad Hussain

Amos Ng

Amr Mohamed Samy Mahdy

Amrit De

Amy O'Shea

An Eng Lim

Ana B. Ruiz

Ana Barbosa

Ana Bela Cruzeiro

Ana Breda

Ana Fialho

Ana Jurinjak Tusek

Ana Klobučar

Ana Leonor Silvestre

Ana M. Portillo

Ana Rosales-Pérez

Ana-Maria Zamfir

Anand Nayyar

Anandakumar Haldorai

Anantachai Padcharoen

Anar T. Assanova

Anas Masarwa

Anastasiya E. Runnova

Anatolij K. Prykarpatski

Anatoliy Prykarpatsky

Anatoliy Swishchuk

Anatoly A. Alikhanov
Anatoly Lisnianski

Anatoly Peresetsky

Anca Avram

Anca Christine Pascu

Anca Croitoru

Anca Sipos

Anda Olteanu

András Mihály

André B. M. Souza

Andre Castro

André Furtado

André Leroy

André Ottoni

Andre Reznikov

Andrea Burlon

Andrea Čajková

Andrea Caporale

Andrea Cioncolini

Andrea Colagrossi

Andrea Flori

Andrea Hawkins-Daarud

Andrea Paltrinieri

Andrea Rosová

Andrea Sambri

Andrea Scapellato

Andrea Schaffer

Andrea Sujova

Andrea Tangherloni

Andrea Tick

Andreas Arvanitoyeorgos

Andreas C. Georgiou

Andreas Henrici

Andreas Holzinger

Andreas Humpe

Andreas Kanavos

Andreas Kreisel

Andreas Makrides

Andreas Neuenkirch

Andreas Philippou

Andreas Rokopanos

Andreea Fulga

Andreea Koreanschi

Andrei C. Holman

Andrei Halanay

Andrei Horvat-Marc

Andrei Karatkevich

Andrei Pogrebkov

Andrei S. Rodin

Andrej Novak

Andrej Sarjas

Andrej Srakar 
Andrés Aragoneses

Andrés Carrión

Andrés Julián Saavedra Montes

Andrew B. Martinez

Andrew Barron

Andrew Gratien Johonpillai

Andrew James Bruce

Andrew Rees

Andrew S. Manikas

Andrew V. Knyazev

Andrew White

Andrey Cherstvy

Andrey Krauklis

Andrey Lychev

Andrey Morgulis

Andrey S. Vasiliev

Andrey Sarikov

Andrey Zahariev

Andrii Shekhovtsov

Andrius Dzedzickis

Andrius Grigutis

Andriy V. Zagorodnyuk

Andronick Aramovich Arutyunov

Andronikos Paliathanasis

Andrzej Chydziński

Andrzej Dzieliński

Andrzej Gnatowski

Andrzej Katunin

Andrzej Łebkowski

Andrzej Raszkowski

Andrzej Świerniak

Andrzej Sztando

Andrzej Tomporowski

Andrzej Tomski

Andrzej Wiśnicki

Aneta Ptak-Chmielewska

Anetta Szynal

Ángel Alberto Magreñán

Ángel De La Torre

Angel Gutierrez

Ángel Javier Aguirre

Angel Luis Muñoz Castañeda

Angel Martin Del Rey

Angel Plaza

Angela Mertig

Angela R. Crawford

Angela Repanovici

Angela Ricciardello

Angela Slavova

Angelica Lo Duca

Angelica Malaspina
Angelo Favini

Angelo Liseno

Angelo Sifaleras

Anh-Khoa Vo

Aniello Buonocore

Anirban Mondal

Anita Habók

Anju Panwar

Anna Baccaglini-Frank

Anna Bryniarska

Anna Drzewicz

Anna Kocira

Anna Łyczkowska-Hanćkowiak

Anna M. Rakoczy

Anna Maria Fino

Anna Maria Fiori

Anna Pierri

Anna Pietrenko-Dabrowska

Anna Rita Sambucini

Anna Saniuk

Anna Schuhbauer

Anna Tirpakova

Anna Tur

Anna Warchlewska

Anna Zielińska-Chmielewska

Annalisa Iuorio

Annarita Colasante

Annarosa Serpe

Anne B. Koehler

Anne Hofmeister

Annunziata Palumbo

Anouar Ben Khalifa

Anouar Ben Mabrouk

Antanas Laurincikas

Antanas Žilinskas

Anthony Aidoo

Anthony J. Guttmann

Anthony Perez

Anthony Rossiter

Anthony Shannon

Anton A. Kutsenko

Anton Antonov

Anton Chepurnenko

Anton Hamler

Anton Iliev

Anton Kasprzhitskii

Anton Miglo

Anton Rassõlkin

Anton Savin

Anton Trushechkin

Antonela Toma 
Antonella Falini

Antonello Rosato

Antonin Dvorak

Antonin Jancarik

Antonín Slavík

Antonin Vitecek

Antonino Galati

Antonino Maniaci

Antonino Polimeno

António Abreu

Antonio Agresta

Antonio Araujo

Antonio Arriaza

Antônio Augusto Fröhlich

Antonio Bernardo-Sánchez

Antonio Boccuto

Antonio Bolufé-Röhler

Antonio Caggiano

Antonio Carozza

Antonio Coelho

Antonio Concilio

Antonio Di Crescenzo

Antonio Falco

Antonio Gloria

Antonio González

Antonio Jiménez-Martín

Antonio López Jaimes

António M. Lopes

Antonio M. Puertas

Antonio Manuel Vargas

Antonio Masiello

Antonio Moreno Verdejo

Antonio Orlandi

Antonio Pacifico

Antonio Partal Ureña

Antonio Pérez Hernández

Antonio Salmerón

Antonio Sánchez-Bayón

Antonios Andreatos

Antoniu Turcu

Antti Lehikoinen

Anuar Ishak

Anuj Sharma

Anurag Jayswal

Anurag Shukla

Anwar Zeb

Anwarud Din

Anyela Valentina Camargo Rodríguez

Apostolos Korlos

Apostolos Syropoulos

Apu Kumar Saha
Apurna Ghosh

Aragonés-Beltrán Pablo

Aravind Sukumaran Rajam

Arbnor Pajaziti

Archibong Archibong-Eso

Ardashir Mohammadzadeh

Areeya Chantasri

Aref Jeribi

Ari Happonen

Ariana Pitea

Arie Wahyu Wijayanto

Ariel Mitev

Arif Hussain

Arif Mandangan

Arif Salimov

Arka Datta

Arkadiusz Jędrzejewski

Arkadiusz Kawa

Arkadiusz M. Kowalski

Arkadiusz Poplawski

Arkady Leiderman

Arlindo Silva

Arman Nedjati

Arman Oshnoei

Arman Shojaei

Armen Sergeev

Armin Varmaz

Arnau Mir Torres

Arnaud Z. Dragicevic

Arne Johannssen

Arpan Kumar Pradhan

Arran Fernandez

Arsalan Najafi

Arsen Palestini

Arsen V. Pskhu

Artamonov Viacheslav Alexandrovich

Artem Denisov

Artur Blaszczuk

Artur Dabrowski

Artur Iluk

Artur Karimov

Artur Kobus

Artur Władysław Ganczarski

Artur Zbiciak

Arturas Kaklauskas

Artūras Kilikevičius

Arturo Hidalgo

Arturo Leccadito

Arturo Tagliacozzo

Arun Arjunan

Arunodaya Raj Mishra 
Arzu Akbulut

Asad Ali Khan

Asaithambi Asai

Asep K. Supriatna

Ashish Rauniyar

Ashkan Almasi

Ashkan Ghanbarzadeh-Dagheyan

Ashley Emery

Ashok Kumar Bagha

Ashraf Khalil

Ashraf Mohamed Tawfik

Ashwin Vinod

Asif Iqbal Malik

Aslina Baharum

Assadullah Khan

Asunción M. Mayoral

Aswi Aswi

Asya Aminova

Atanas Ivanov

Atanaska Georgieva

Atanu Chatterjee

Atef Hobiny

Athanasios A. Pantelous

Athanasios Anagnostis

Athanasios G. Georgiadis

Athanasios Karlis

Athanasios Nikolaidis

Athanasios Tsagkanos

Athanasios Vasilakos

Atta Ur Rehman

Atthaphon Ariyarit

Attila Egri-Nagy

Attila Gulyás

Attila Gyulassy

Audrius Čereška

Audrius Kabasinskas

Augustín Stareček

Augustine O. Nwajana

Augusto Ferrante

Augustyn Lorenc

Aurelio Fernández Bariviera

Avrilia Konguetsof

Awais Younes

Aydin Azizi

Ayeley Tchangani

Azadeh Zahedi Khameneh

Azhar Ali Zafar

Azita Manouchehri

Azra Tafro

B. C. Prasannakumara

Babak Shiri
Babu R. Dawadi

Bahman Moraffah

Bahram Sadeghi Bigham

Balaji Selvarajou

Balan Sundarakani

Balázs Égert

Balázs Kocsi

Balazs Toth

Balbina Casas-Méndez

Baodi Liu

Baoling Zou

Baowei Wang

Bapan Ghosh

Barath Narayanan

Barbara Calabrese

Barbara Cardone

Barbara Sabitzer

Barbara Schulte

Baris Baykant Alagoz

Barman Nabajeet

Bartłomiej Ambrożkiewicz

Bartłomiej Jefmański

Bartłomiej Kizielewicz

Bartolomeo Silvestri

Bartosz Fikus

Bartosz Naskręcki

Bartosz Sawik

Basem Aref Frasin

Bashir Ahmad

Basil Papadopoulos

Bassem Abd-El-Atty

Batmend Horoldagva

Batrancea Ioan

Batyr Orazbayev

Bayu Adhi Tama

Beata Mrugalska

Beatriz Molina Serrano

Begoña Esther Sampedro

Behnam Tavakkol

Behnam Tonekaboni

Behnam Yazdani Bejarbaneh

Behzad Farahani

Behzad Vaferi

Béla Takarics

Belayat Hossain

Belén Muñoz

Belmiro Duarte

Belov Aleksei Yakovlevich

Belov Alexei Yacovlevich

Ben Andrews

Ben Levy 


\author{
Ben Lutz \\ Ben Strasser \\ Benedek N. Nagy \\ Benjamin Ricaud \\ Benjamin Wacker \\ Benjamin Yen \\ Benoit Morel \\ Beny Neta \\ Benyamin Ahmadnia \\ Beorchia Valentina \\ Berinderjeet Kaur \\ Berislav Žmuk \\ Berna Kiraz \\ Bernard B. Munyazikwiye \\ Bernardo B. Gatto \\ Bernardo D'Auria \\ Bernardo Spagnolo \\ Bertrand Tavin \\ Bessem Samet \\ Beverly Dawn Metcalfe \\ Bhanwar Lal Puniya \\ Bharat Sarath \\ Bharath Babu Nunna \\ Bharath Nagaraja \\ Biagio Simonetti \\ Bianca Caiazzo \\ Biancamaria Della Vecchia \\ Bilal Bilalov \\ Bin Chen \\ Bin Zhang \\ Bin Zou \\ Bingchen Liang \\ Bingyang Cao \\ Bingyang $\mathrm{Hu}$ \\ Binh Nguyen \\ Binqiang Chen \\ Biswajit Sarkar \\ Biswarup Bhattacharyya \\ Björn Gustafsson \\ Björn Krüger \\ Blaise Bourdin \\ Blanka Horvath \\ Blanka Tundys \\ Blaž Likozar \\ Błażej Mazur \\ Bo Liu \\ Bo Markussen \\ Bo Tian \\ Bo Wang \\ Boca Gratiela Dana \\ Bocheng Jin
}

Boda Ning

Bodo Herzog

Bogdan Căruntu

Bogdan Iancu

Bogdan Lipuš

Bogdan Marinca

Bogdan Tiganoaia

Bogdán Zaválnij

Bohdan Datsko

Bo-Lin Jian

Bonifacio Llamazares

Boris Aguilar

Boris Andrievsky

Boris Goldfarb

Boris Hasselblatt

Boris Malomed

Boris Mikhailovich Shumilov

Boris Ryabko

Borislav Bedzhev

Borislav Stoyanov

Borivoje Pašić

Borja Espejo-García

Borna Abramovic

Bosiljka Tadic

Boško Damjanović

Boštjan Kovačić

Boštjan Šumak

Boussad Abbes

Boutalis Ioannis

Bowen Huang

Boyan Dimitrov

Boyana Norris

Bozidar Ivankovic

Brad Merrick

Branislav Randjelovic

Brett Bligh

Brian Ingalls

Brian Kelley

Brian McSkimming

Brian R. Williams

Brian Vant-Hull

Brianna Kurtz

Brij Gupta

Brodie Lawson

Bruce Hartman

Bruce Morley

Brunero Liseo

Bruno Venditto

Bruno Viscolani

Bugra Alkan

Bülent Delibas 
Bumned Soodchomshom

Burak Erkut

Burcu Gürbüz

Byeong Yong Kong

Byungeun Pak

Byung-hoon Kim

C. Balsa

C. Ravichandran

Caglar Karamasa

Cai-Wan Chang-Jian

Calin Ciufudean

Calin Vaida

Calin-Adrian Comes

Calin-Ioan Gheorghiu

Calogero Vetro

Calvo Maria Luisa

Camelia Chira

Camelia Daniela Hategan

Camelia Delcea

Camelia Oprean-Stan

Camelia Petrescu

Camelia-M. Pintea

Can Nerse

Canh-Dung Tran

Canjun Yang

Canrong Tian

Cao Wang

Capperucci Davide

Carina Geldhauser

Carl Strathearn

Carla Pinto

Carlo Cafaro

Carlo Fantozzi

Carlo Gualtieri

Carlo Sanna

Carlos Alberto De Bragança Pereira

Carlos David Zuluaga

Carlos Grilo

Carlos Hervás-Gómez

Carlos J. Pérez-González

Carlos Kubrusly

Carlos M. Fernandes

Carlos Martínez Von Dossow

Carlos Molina

Carlos Moreno-García

Carlos Rocha

Carlos Romero

Carlos Sánchez-López

Carmelo Majorana

Carmelo Scuro

Carmen Chicone
Carmen De Pablos

Carmen Del Pilar Gallardo Montes

Carmen Gale

Carmen García Centeno

Carmen León-Mantero

Carmen Monica Păunescu

Carmen Moret-Tatay

Carmen Patino-Alonso

Carol Hargreaves

Carol Nash

Caroline König

Carsten Hartmann

Casper Agaton

Catalin I. Pruncu

Catalin Popescu

Catalin Stoean

Catalina Bolancé

Catarina Afonso Alves

Catherine Dezan

Cecília Szigeti

Cees A. Swenne

Cees Diks

Célio Fernandes

Cemil Tunc

Cenk Çalışkan

Ce-Zhong Tong

Ch. Tsitouras

Chabi Rani Panigrahi

Chairi Kiourt

Chandan Pandey

Chandler C. Benjamin

Chandra Pappu

Chandramohan Dhasarathan

Chang Jiang Wang

Chang Liu

Chang-Beom Choi

Changbum Chun

Changchun Liu

Chang-Gyun Roh

Changhwa Woo

Chanyoung Park

$\mathrm{Chao} \mathrm{Fu}$

Chao Huang

Chao Wang

Chao Zhang

Chaojie Li

Charalampos Siristatidis

Charis Ntakolia

Charles Dunkl

Charles Madewell

Charles Reichhardt 
Charles Sutcliffe

Chee Wei Ang

Chemseddine H. Mâatki

Chen Fulong

Chenchu Xu

Cheng Wang

Cheng-Fu Huang

Cheng-Jie Liu

Chenglong Shao

Chengqing Li

Cheon Won Choi

Cheong K. Park

Cheonshik Kim

Cherng-tiao Perng

Chetan Kumar Vudadha

Chetna Gupta

Cheung-Chieh Ku

Chi Wah Kok

Chia-Hsiang Lin

Chiara Bedon

Chiara Mocenni

Chia-Wei Lee

Chien Wern

Chien-Chung Chen

Chih-Hong Lin

Chih-Te Yang

Chi-Hua Chen

Chih-Wen Chang

Chihwen Hsueh

Chih-Yu Liu

Chih-yuan Tseng

Ching Sing Chai

Ching-Hsin Wang

Ching-Lueh Chang

Chin-Ling Chen

Chin-Shiuh Shieh

Chinthaka Premachandra

Chokri Kooli

Chong Mun Ho

Chris G. Karayannis

Chris G. Tzanis

Christian A. Rivera

Christian Bongiorno

Christian D. Chapman

Christian Genest

Christian Heumann

Christian Kunde

Christian Napoli

Christian Offen

Christian Siatka

Christian Weis
Christine Mwase

Christodoulos Sophocleous

Christoforos L. Raptopoulos

Christoph Bayer

Christoph Bühren

Christoph Frei

Christoph Kern

Christoph S. Weber

Christoph Steup

Christophe Chalons

Christophe Chesneau

Christophe Ringeissen

Christopher Douglas

Christopher K. Merker

Christopher Martin Van Bommel

Christopher W. Curtis

Christos Floros

Christos G. Massouros

Christos Katris

Christos Kountzakis

Christos Sourdis

Christos Tantos

Christos Troussas

Chrysanthos Maraveas

Chuan Li

Chuanxi Zhu

Chun Ki Chan

Chunbiao Li

Chung Kwan Lo

Chung-Chuan Chen

Chunho Yeom

Chunhong Zhao

Chun-Min Yu

Chun-Wei Yang

Chunyu Wang

Chuong V. Nguyen

Chyan-Long Jan

Chyi Lin Lee

Ci Jian Yang

Cihan Bayindir

Cihan Özgür

Ciprian Lupu

Ciprian Orhei

Clara Grácio

Clara Ionescu

Claudia Ceci

Claudia Giacomozzi

Claudia Marzi

Claudia Pacurar

Claudia Stoian

Claudio Macci 
Claus Fieker

Claus H. Carstensen

Clemens Fuchs

Clemente Cesarano

Codruta Stoica

Concetta Pirrone

Concettina Buccella

Cong Qi

Conghua Wen

Congxu Zhu

Conrado Miguel Manuel García

Constantin Aurelian Ionescu

Constantin Fetecau

Constantin Ilie

Constantin Udriste

Constantin Volosencu

Constantinos Petropoulos

Cornelia Gyorodi

Cornelia-Livia Bejan

Cornelie Leopold

Cornelis W. Oosterlee

Corrado Lo Storto

Corrine Warren Ruktanonchai

Cory Howk

Cory McKay

Cosimo Ieracitano

Cosimo Lupo

Coşkun Çetin

Cosmin Anitescu

Cosmin Sabo

Costa Eduardo

Costanza Conti

Costel Plescan

Craig Carpenter

Crispin M. Mutshinda

Cristea Irina

Cristescu Gabriela

Cristi Spulbar

Cristian Busu

Cristian Lazureanu

Cristian Stătescu

Cristiana Ionescu

Cristiana Silva

Cristina Campi

Cristina Caridade

Cristina Cirtoaje

Cristina Dumitriu

Cristina Flaut

Cristina Georgiana Zeldea

Cristina Martínez

Cristina Muresan
Cristina Nicolau

Cristina Raluca Gh. Popescu

Cristina Serpa

Cristina Sousa

Cristina Voicu

Cristina-Elena Hretcanu

Csaba Farkas

Csaba Levente Nagy

Csaba Sidor

Csaba Szabó

Cuauhtémoc Sanchez Ramírez

Cun Ji

Cynthia Tapia

Cyril Fischer

Czesław Dyrcz

Czesław Mesjasz

D. D. McNutt

Dachun Yang

Dae-Eun Lim

Daekook Kang

Dafang Zhao

Dagmar Čámská

Dagmar Medková

Daiqiang Zhang

Daisuke Murakami

Daisuke Yoshikawa

Daiva Jureviciene

Dalia Kriksciuniene

Daliang Zhao

Dalibor Gonda

Dalin Zhang

Damian Gogolewski

Damian Słota

Damian Wierzbicki

Damiano Torre

Damir Jukić

Damir Kinzebulatov

Dan Chicea

Dan Ioan Topor

Dan Kucerovsky

Dan Lascu

Dan Li

Dan Nitoi

Dan Selisteanu

Dan Vilenchik

Dana Badau

Dana Dannélls

Dana Smetanová

Dana-Mihaela Petroșanu

Dancho Petrov

Dan-Cristian Popa 
Dani Moreno

Danica Rosinová

Daniel A. Griffith

Daniel Bearup

Daniel Bochsler

Daniel Breaz

Daniel Caballero-Julia

Daniel Condurache

Daniel De La Fuente

Daniel Gaigall

Daniel Homocianu

Daniel J. F. Fox

Daniel Jardon

Daniel Kaminski

Daniel Lepadatu

Daniël Linders

Daniel Papla

Daniel Peralta

Daniel Sanchez-Taltavull

Daniel Sepúlveda

Daniel Sevcovic

Daniel W. K. Tse

Daniel Zhengyu Huang

Daniela Marian

Daniela Roșca

Daniela Zaharie

Daniela-Elena Popescu

Daniele Cafolla

Daniele Ritelli

Daniele Tommasini

Daniel-Ioan Curiac

Danielli Araújo Lima

Danijel Grahovac

Danijela Rabar

Danijela Tuljak-Suban

Danilo Capecchi

Danilo Costarelli

Danilo D'Andrea

Danilo Sartorello Spinola

Daniyal Haider

Danko D. Georgiev

Danko Radic

Danny Barash

Danuta Szpilko

Daowen Qiu

Daria Bylieva

Darin J. Ulness

Dario Fasino

Dário Ferreira

Dario Maimone Ansaldo Patti

Darío Ramos-López
Darius Siauciunas

Dariusz Horla

Dariusz Krzywda

Dariusz Mikielewicz

Dariusz Plinta

Dariusz Puchala

Dariusz Rzońca

Dariusz W. Brzeziński

Darja Rupnik Poklukar

Darjan Karabasevic

Darko Božanić

Darko Vukovic

Darren A. Narayan

David Almorza Gomar

David Bassir

David Cabaleiro

David Coker

David González-Gómez

David Greiner

David Hill

David J. Jeffrey

David J. Kukulka

David J. Olive

David Jesenko

David Juárez Varón

David Lane

David M. Dueber

David Müller-Bender

David Nash

David Oaknin

David Quero

David Rothschild

David Ruiz Baños

David T. Yost

David Vidal-Tomás

Davide Astolfi

Davide Cinquegrana

Davide Guido

Davide Penazzi

Davide Proserpio

Davide Spinello

Davut Izci

Dawar Khan

Dawid Połap

Dawit Aberra

Da-Zhi Sun

Dean Fantazzini

Debasmita Basu

Debayan Dey

Debisree Ray

Debkalpa Goswami 
Deborah Konkowski

Debsindhu Bhowmik

Decky Aspandi

Deepak Nag Ayyala

Degan Zhang

Dehai Kong

Demian Antony D'Mello

Deng Wang

Denis Butusov

Denis Horvath

Denis Krotov

Denis N. Sidorov

Denis Sabirov

Denis Težak

Denis Tihon

Denis Zakharov

Deniz Cetinkaya

Dennis Belomestny

Deog Jae Hur

Deolinda M. L. Dias Rasteiro

Der-Chyuan Lou

Derek Young

Der-Ming Ma

Desirée Joosten-ten Brinke

Despoina I. Makrygiorgou

Destiny E. O. Anyaiwe

Devanjan Bhattacharya

Devaraja Naik

Devender Kumar Saini

Dewey Taylor

Dezhou Guo

Dharmendra Kumar

Di Wang

Diana Berbecaru

Diana Kalibatiene

Diana Mindrila

Diana Otrocol

Diana Rokita-Poskart

Diana Savin

Diane Peurichard

Didier G. Leibovici

Didier Trouche

Diego Gallardo

Diego Marques

Diego Misseroni

Diego Vergara

Dieter Claeys

Dillip Kumar Das

Dilna Nataliya

Dima V. Valovik

Dimiter Prodanov
Dimitra Karagkouni

Dimitri Pétritis

Dimitrios E. Koulouriotis

Dimitrios G. Koubogiannis

Dimitrios Konispoliatis

Dimitrios Mitsotakis

Dimitrios Nalmpantis

Dimitrios Nikolopoulos

Dimitrios P. Sokolis

Dimitrios Pyromalis

Dimitrios Stamovlasis

Dimitrios Vezeris

Dimitris Christodoulou

Dimitris Georgiou

Dimitris Kallioras

Dimitris Kamilis

Dimitris Karampatzakis

Dimitris Kenourgios

Dimitris Kilakos

Dimitris Tselios

Dimitris Zissis

Dina Ibrahim

Dinara Sobola

Dinesh Kumar

Dinesh Kumar Sharma

Dinh Van Nam

Dinh-Thuan Do

Diogo Poças

Dionisio F. Yáñez Avendaño

Dionisis Stefanatos

Dipak Kumar Mandal

Dipankar Roy

Dipayan Guha

Dirk Thorleuchter

Diyi Chen

Dmitri E. Kvasov

Dmitrii Kaplun

Dmitrii Karp

Dmitrii Legatiuk

Dmitrij I. Nagirner

Dmitriy V. Ivanov

Dmitry Efrosinin

Dmitry Ermakov

Dmitry Kovalevsky

Dmitry Krushinsky

Dmitry Lukyanenko

Dmitry Makarov

Dmitry Mikhailovich Nazarov

Dmitry Pavlyuk

Dmitry Ponomarev

Dmitry Roldugin 
Dmitry Ruban

Dmitry Sokoloff

Dmitry V. Balandin

Dmitry V. Kruchinin

Dmytro Osiichuk

Dobrotă Gabriela

Dokyun Kim

Dolores Gallardo Vázquez

Domenico Albano

Domenico Brunetto

Domenico Ciuonzo

Domenico Curto

Domenico Freni

Domenico Guida

Domenico Montemurro

Domenico Palladino

Domenico Pascarella

Domenico Tallarico

Domenico Vitulano

Domingo Barrera Rosillo

Domingo Benitez

Domingo Gómez Pérez

Dominik Endres

Dominik Tomaszuk

Dominik Zimon

Dominika Dabrowska

Dominique Estival

Dominique Popescu

Don Hong

Don Koks

Donald DeAngelis

Dong Huang

Dong Yun Shin

Dongfang Li

Dong-Her Shih

Dong-Hyuk Im

Dongkyu Lim

Dongming Chen

Dongxu Liu

Dongyi Wang

Doo Heon Song

Dor Abrahamson

Dora Pokaz

Dorian Cojocaru

Dorian Sidea

Dorian Skrobek

Dorijan Radočaj

Dorin Maier

Dorina Clichici

Dorina Raducanu

Dorota Żebrowska-Suchodolska
Douadi Drihem

Douglas Anderson

Douglas B. Meade

Douglas J. Hodgson

Douglas O'Shaughnessy

Doutora Sónia Rolland Sobral

Dovì Vincenzo

Dragan Marinkovic

Dragan Pamučar

Dragoljub Bajić

Dragoljub Gajic

Dragos-Patru Covei

Dragos-Victor Anghel

Dražen Novaković

Duarte Valério

Duc Duy Nguyen

Duksan Ryu

Dulani Meedeniya

Dumitrescu Catalin

Dumitru Baleanu

Dumitru Motreanu

Dumitru Vieru

Dumitru-Clementin Cercel

Dunhui Xiao

Durga H. Kutal

Dusan Jandacka

Dusan Milosevic

Dusan Rakic

Dwueng-Chwuan Jhwueng

E. L. Shishkina

E. Laxmi Lydia

E. Providas

Eabhnat Ni Fhloinn

Ebenezer Atta Mills

Ebrahim Ghaderpour

Ebrahim Ghasemi

Ecaterina Daniela Zeca

Eckhard Hitzer

Edgar Erdfelder

Edgar Solomonik

Edi Kurniawan

Edmondo Minisci

Edmondo Trentin

Edmundas Kazimieras Zavadskas

Edmundo Huertas

Edoardo Ballico

Edoardo Saccenti

Edouard Ivanjko

Eduard Baitinger

Eduard S. Sopin

Eduard Zadobrischi 
Eduardo Cabal-Yepez

Eduardo Corral Abad

Eduardo J. S. Villaseñor

Eduardo M. Gouveia

Eduardo Quevedo-Gutiérrez

Edward Preweda

Edward S. Krebes

Edyta Kardas

Edyta Plebankiewicz

Effie Marcoulaki

Efstathios Magerakis

Efstratios Emm. Tzirtzilakis

Ehab Essa

Ehsan Harirchian

Ehsan Naderi

Ehsan Nazemi

Eirini Eleni Tsiropoulou

Ekaterina Orlova

Elaheh Yadegaridehkordi

Eleftherios Tsakirakis

Elena Basan

Elena Boguslavskaya

Elena Butsenko

Elena Castro-Rodríguez

Elena Cichero

Elena Druica

Elena Ferretti

Elena Gorbashko

Elena Gregova

Elena Gubar

Elena Korchagina

Elena Nechita

Elena Niculina

Elena Parilina

Elena Simina Lakatos

Elena Solovyeva

Elena Untaru

Elena-Corina Cipu

Eleni Makri

Eleni Zafeiriou

Elham Bahmanteymouri

Eliana Costa e Silva

Elias Dritsas

Elías Escobar-Gómez

Elias Roumpakias

Elio Chiodo

Elisa Alòs

Elisa Cabana

Elisabete Alberdi Celaya

Elisabete Félix

Elisan Dos Santos Magalhães
Elizabeth Behrman

Elizabeth Hunter

Elkhan Richard Sadik-Zada

Elmer Rolando Llanos Villarreal

Elnaz Rezaian

Elodie Pahon

Elsayed Badr

Elvira Barbera

Elvira Fernández-Ahumada

Elvira Romano

Elzbieta Jasinska

Elżbieta Macioszek

Elzbieta Sidorowicz

Elżbieta Sobczak

Elżbieta Szczepankiewicz

Emad A. Mohamed

Emanuel Guariglia

Emanuel Weitschek

Emanuela Rosazza Gianin

Emanuele Quaranta

Emanuele Taufer

Emanuele Zappala

Emel Aktas

Emese Beáta Berei

Emil Molnar

Emil Saucan

Emilia Bazhlekova

Emília Bigotte De Almeida

Emilia Florio

Emilia Herman

Emilia López-iñesta

Emilian Szczepański

Emiliano Sironi

Emilio Abad-Segura

Emilio Acerbi

Emilio Cano

Emilio Congregado

Emilio Cruciani

Emilio Matricciani

Emily Stone

Emin Bayraktar

Emma Chiaramello

Emma Previato

Emmanuel Kwasi Mensah

Emőke-Szidónia Feder

Emre Demirkaya

Endre Kovács

Endre Pap

Enikő Kácsor

Ennio Bilancini

Enrica Pirozzi 


\author{
Enrica Santucci \\ Enrico De Micheli \\ Enrico Feoli \\ Enrico Ferrentino \\ Enrico Ivaldi \\ Enrique Carmona Medeiro \\ Enrique Jimenez-Rodríguez \\ Enrique Miranda \\ Enrique $\mathrm{Mu}$ \\ Enrique Peacock-López \\ Enrique Sánchez Pérez \\ Eoin Plant \\ Ercan Kose \\ Erfan Shojaei Barjuei \\ Erhan Güler \\ Eric Florentin \\ Eric Nyarko \\ Eric Rombokas \\ Eric S. Kawaguchi \\ Eric S. Weber \\ Eric T. Chung \\ Eric Weisbrod \\ Erik Behringer \\ Erik Kučera \\ Erkinjon Karimov \\ Ern Gun Kwon \\ Ernesto Aranda-Escolástico \\ Ernesto Colomo Magaña \\ Ernesto Rodríguez-Crespo \\ Ernesto Zambrano-Serrano \\ Esmail Peyghan \\ Esmeralda Maina \\ Esra Akbas \\ Esteban Jove \\ Esteban Rougier \\ Esther Ramírez \\ Etienne Boileau \\ Etienne E. Kerre \\ Eugen Anitas \\ Eugene Postnikov \\ Eugene Semenkin \\ Eugenia Grecu \\ Eugenio Roanes-Lozano \\ Eugeniusz Gatnar \\ Eulalia Martínez Molada \\ Eulalia Nualart \\ Eun-jin Kim \\ Eva Boj Del Val \\ Eva H. Dulf \\ Éva Kaslik \\ Eva Trojovská
}

Evangelos Bekiaris

Evangelos Tyflopoulos

Evelin Krmac

Evgenii Khailov

Evgenii Prosviryakov

Evgenii S. Baranovskii

Evgeniya Pankratova

Evgeny A. Antipov

Evgeny Gordon

Evgeny M. Mirkes

Evgeny Nikulchev

Evgeny Ryzhov

Evgeny Semenovich Zhukovskiy

Evgueni Doubtsov

Evizal Abdul Kadir

Evrencan Ozcan

Evsey Morozov

Ewa Drgas-Burchardt

Ewa Dziwok

Ewa Korzeniewska

Ewa Roszkowska

Eze Raymond Nwaeze

Ezio Venturino

Ezzatallah Baloui Jamkhaneh

F. J. Lobillo

F. Muge Sakar

Fabian Scheipl

Fabien Besnard

Fábio Albuquerque

Fábio Azevedo

Fabio Camilli

Fabio Caraffini

Fabio D'Andreagiovanni

Fabio Dercole

Fabio Di Cosmo

Fabio Di Nunno

Fabio Di Troia

Fabio Durastante

Fábio Fernandes

Fabio Maggio

Fabio Podestà

Fabio Rapallo

Fabio Rinaldi

Fabio Rizzo

Fabio V. Difonzo

Fabiomassimo Mango

Fabrizia Guglielmetti

Fabrizio Balducci

Fabrizio Maturo

Fabrizio Scozzese

Fadi Muhediat 


\author{
Fahad Al Basir \\ Fahad Albalawi \\ Fahed Alkhabbas \\ Fahimeh Nazarimehr \\ Faïçal Ndaïrou \\ Faisal Jamil \\ Faizan Ahmed \\ Fan Yang \\ Fang-Bao Tian \\ Fangfang Zhang \\ Fanhai Zeng \\ Faranak Rabiei \\ Fardin Khalili \\ Farhad Jafari \\ Farman Ali \\ Farnoosh Abbas-Aghababazadeh \\ Farooq Ahmad \\ Farooq Gujar \\ Farrah Kristel Batista \\ Farrukh Maksutovich Mukhamedov \\ Faruk Karaaslan \\ Faruk Özger \\ Farzad Kiani \\ Farzan Akbaridoust \\ Farzan Majeed Noori \\ Farzin Piltan \\ Fateh Mebarek-Oudina \\ Fatemeh Rahmani \\ Fatih Yilmaz \\ Fátima Leal \\ Fatima Salahdine \\ Fatmawati Fatmawati \\ Fatos Xhafa \\ Fausto Montoya \\ Fayçal Hammad \\ Federico Pagnozzi \\ Federico Papa \\ Fedor Duzhin \\ Fei Qin \\ Fei Xu \\ Feifei Du \\ Feifei Jin \\ Felipe Martins Muller \\ Felix Arion \\ Felix Dietrich \\ Felix Mauersberger \\ Felix Sadyrbaev \\ Feng Chen \\ Feng Wen \\ Feng Yu \\ Fengming Li
}

Ferda Halicioglu

Ferdinando Di Martino

Ferdinando Zullo

Ferhat Ozgur Catak

Fernanado Barbero

Fernanda Botelho

Fernanda Cristina Corrêa

Fernando Antoñanzas

Fernando Castano Romero

Fernando Etayo

Fernando L. Pelayo

Fernando Marmolejo-Ramos

Fernando Reinaldo Ribeiro

Fernando Sánchez Lasheras

Fernando Tohme

Ferruccio Guidi

Filipe J. Marques

Filippo Gazzola

Filippos Giannakas

Filomena Di Tomasso

Firdous A. Shah

Firooz A. Sadjadi

Fivos Papadimitriou

Flavio Boccia

Fleurianne Bertrand

Florenc Demrozi

Florent Sureau

Florentino Borondo

Florian Bourse

Florian Ion T. Petrescu

Florian Munteanu

Florin Avram

Florin Constantin Mihai

Florin Leon

Florin Munteanu

Florin Nichita

Florin Valentin Rusca

Florina Virlanuta

Fong-Zuo Lee

Fotini Konstantakopoulou

Fouad Jawab

Francesc Font

Francesc Sebé

Francesca Brini

Francesca Magionesi

Francesca Pitolli

Francesco Aymerich

Francesco Bertocci

Francesco Carlo Morabito

Francesco Clementi

Francesco De Giovanni 
Francesco Dell'Accio

Francesco Dell'Isola

Francesco Gargano

Francesco Isaia

Francesco Mainardi

Francesco Marino

Francesco Montefusco

Francesco Nicassio

Francesco Oliveri

Francesco Pascale

Francesco Pediconi

Francesco Regazzoni

Francesco Rundo

Francesco Sica

Francesco Tajani

Francesco Tornabene

Francesco Villecco

Francescomaria Faticanti

Francielle Santo Pedro

Francisco Ascui

Francisco Bernal

Francisco Cruz

Francisco Fernández-Navarro

Francisco German Badia

Francisco Gonzalez-Longatt

Francisco Gutiérrez

Francisco Haces Fernandez

Francisco J. Navarro

Francisco Jareño

Francisco Javier Cárcel-Carrrasco

Francisco Javier Garcia-Pacheco

Francisco Javier Santos-Arteaga

Francisco José Vázquez Polo

Francisco Jurado

Francisco Martínez González

Francisco Milán López

Francisco Navarro Valverde

Francisco P. Romero

Francisco Pérez-Hernández

Francisco Rego

Francisco Rodríguez

Francisco Salas-Molina

Francisco Torres-Ruiz

Francisco Triguero Ruiz

Francisco Ureña Prieto

Francisco Zamora-Polo

Francisco-Ignacio Revuelta-Domínguez

Franco Bagnoli

Franco Zivcovich

Francois Gay-Balmaz

Francois Rivet
Francois Schmitt

Francoise Couenne

Françoise Tisseur

François-Éric Racicot

Frank Gomes-Silva

Frank Li

Frank Phillipson

František Zbořil

Fred Espen Benth

Frédéric Ouimet

Frédéric Schneider

Frederick Peck

Frederike Neuber

Fredy Hoyos Velasco

Friedhelm Schwenker

Frode Rønning

Fu Chen

Fu-Gui Shi

Fukang Zhu

FuLin Luo

Fülöp Melinda Timea

Fulvia Pennoni

Fulya Kula

Furqan Aziz

Fyodor Syomin

G. Charles-Cadogan

G. Donald Allen

G. Ducoffe

G. Kanagaraj

G. Muhiuddin

G. N. V. Kishore

Gabi Hanukov

Gábor Szederkényi

Gabriel Bercu

Gabriel Ciobanu

Gabriel Eduard Vilcu

Gabriel Fedorko

Gabriel Katz

Gabriel Prăjitură

Gabriel Pripoae

Gabriel Ruiz-Garzon

Gabriel Salierno

Gabriela Cristescu

Gabriela Ioana Usurelu

Gabriela O. Chiciudean

Gabriela Pavlovičová

Gabriel-Alexandru Constantin

Gabriele Magna

Gabriele Virzì Mariotti

Gabriella Bognár

Gabriella Bretti 
Gabriella Casalino

Gabriella Gigante

Gaetano Giunta

Gaetano Zimbardo

Gagandeep Kaur

Gai-Ge Wang

Galina Bogdanova

Galina Ilieva

Galina Muratova

Ganapati Sahoo

Ganesh Ghorai

Gang Kou

Gang Lei

Gangadharan Murugusundaramoorthy

Gao Wei

Gaorong Li

Gaoxia Zhu

García Fernando

Gareth Baxter

Garfias Macedo

Garik Petrosyan

Garth Isaak

Gastón Márquez

Gaukhar Shaikhova

Gaurav Dhiman

Gaven Martin

Gavril Grebenisan

Gediminas Gaidulis

Gee Lee

Gelayol Golcarenarenji

Gelson J. Pagan-Diaz

Gennadi Malaschonok

Gennadii Demidenko

Gennady Bocharov

Gennaro Infante

Geoffrey Crisp

Geoffroy Chaussonnet

Geonwoo Kim

George A. Papagiannopoulos

George A. Tsihrintzis

George Belgiu

George Chatzarakis

George D. Verros

George Efremidis

George Fikioris

George K. Adam

George Kornaros

George Lazaroiu

George Selvam

George Suciu

George Teseleanu
George Tzougas

Georgeta Soava

Georgi Georgiev

Georgia Ellina

Georgia Irina Oros

Georgios A. Papadopoulos

Georgios Alexandridis

Georgios N. Kouziokas

Georgios Papavasileiou

Georgy Sofronov

Gerald Aranoff

Gerald Cleaver

Gerald H. L. Cheang

Gerald H. Tomkin

Gerald N. Flerchinger

Geraldo Pereira Rocha Filho

Gerardo Catapano

Gerardo Flores

Gerasim Vladimirovich Krivovichev

Gerasimos T. Soldatos

Gerd Christoph

Gerda Ana Melnik-Leroy

Gergő Nemes

Gerhard W. Weber

Gerhard Wellein

Gero Szepannek

Gevork B. Gharehpetian

Geyverson Teixeira De Paula

Ghasem Derakhshan

Gheorghe H. Popescu

Gheorghe Moroșanu

Gheorghe N. Popescu

Gheorghe Nagit

Gheorghe Oros

Gheorghe Săvoiu

Gheorghe Sebestyen

Gherardo Varando

Ghiocel Groza

Ghous Ali

Ghulam Mustafa

Ghulam Rasool

Gia Sirbiladze

Giacomo Ascione

Giacomo Elefante

Giacomo Patrucco

Giada Adelfio

Giada LaScalia

Giambattista Bufalino

Gian Paolo Clemente

Giancarlo Rinaldo

Giancarlo Sperlì 
Gianfranco Forte

Gianluca Faraco

Gianluca Zitti

Gianluigi Greco

Gianluigi Me

Gianmarco Giovannardi

Gianna M. Del Corso

Gianni Arioli

Gianni Betti

Gianni Bosi

Gianni D'Angelo

Gianni Manno

Gianni Pagnini

Giannis Karagiannakis

Gianpaolo Palma

Gianpiero Colangelo

Gianvito Scaringi

Giedre Streckiene

Gijs Schoenmakers

Gil Cohen

Gil Jorge Barros Henriques

Gilbert Lim

Gilberto Bernardes

Gilberto Gonzalez-Parra

Gilberto Medeiros Nakamura

Gilberto Rivera

Gilberto Santos

Gilles Aldon

Gintautas Dzemyda

Gioacchino Alotta

Giordano Santilli

Giorgio Gronchi

Giorgio Gubbiotti

Giorgio Sonnino

Giovana Goretti Feijó De Almeida

Giovanna Bimonte

Giovanni Angiulli

Giovanni Apruzzese

Giovanni Bortolan

Giovanni Campisi

Giovanni Conti

Giovanni De Luca

Giovanni Delnevo

Giovanni Lo Faro

Giovanni Maria Sardi

Giovanni Mummolo

Giovanni Naldi

Giovanni Paragliola

Giovanni Pau

Giovanni Schmid

Giovanni Vinti
Gisela Pujol Vazquez

Gissell Estrada-Rodriguez

Giulia Caruso

Giulia Dileo

Giulia Lisarelli

Giuseppe Barca

Giuseppe Cardone

Giuseppe Dattoli

Giuseppe Jurman

Giuseppe Leonardo Cascella

Giuseppe Mingione

Giuseppe Nasso

Giuseppe Orlando

Giuseppe Ruta

Giuseppe Varone

Giuseppe Viglialoro

Giusppe Attanasi

Giusy Mazzone

Gleb Pogudin

Gloria Cerasela Crisan

Godwin Amechi Okeke

Gongjun $\mathrm{Xu}$

Gongping Huang

Gopal Krishan

Gopi Battineni

Goran Buturac

Goran Cirovic

Gorazd Bombek

Gornushkin Igor

Gowtham Muniraju

Graham Hall

Graziana Cavone

Grażyna Janikowska

Gregor Papa

Gregorio Gimenez

Gregorio Serna

Gregory Francois

Gregory Miller

Grigoras Gheorghe

Grigore Salagean

Grigori Panasenko

Grigorios Zouros

Grigory Panovko

Grover Zurita

Grzegorz Bazydło

Grzegorz Filcek

Grzegorz Filo

Grzegorz Gembalczyk

Grzegorz Grela

Grzegorz Karoń

Grzegorz Lesiuk 
Grzegorz Maciejewski

Grzegorz Mentel

Grzegorz Michalski

Grzegorz Redlarski

Guang Yin

Guangdong Duan

Guangming Zhang

Guangyou Zhou

Guanjun Wang

Guanjun Xu

Guanming He

Guanqiu Qi

Guanyu Piao

Guennady Ougolnitsky

Guglielmo D'Amico

Guido Marseglia

Guilherme Wood

Guillaume Chauvet

Guillaume Metzler

Guillaume Sandou

Guillermo Huerta Cuellar

Guillermo Martínez-Flórez

Guillermo Morales Luna

Guillermo Restrepo

Guiping $\mathrm{Hu}$

Gulasekaran Rajaguru

Günay Öztürk

Günter Bärwolff

Gunvant Achutrao Birajdar

Guoju Ye

Guojun $\mathrm{Yu}$

Guoqi Ma

Gurami Shalvovich Tsitsiashvili

GuruPrasad Samanta

Gustavo Adolfo Muñoz

Gustavo Filipe Pinto

Gustavo Pelaitay

Gustavo Raush

Guy Mélard

Gwo-Jen Hwang

György Dósa

Gyorgy Gyorok

Gyula Gróf

Gyurhan Nedzhibov

H. T. Liu

Haci Mehmet Baskonus

Hadeed Ahmed Sher

Hadi Alizadeh Noughabi

Hadi Hasanzadehshooiili

Haewon Byeon

Hafiz Suliman Munawar
Hai Wang

Haider Ali

Haider Al-Khateeb

Haifeng Wang

Haigang Sui

Haijun Yu

Haim Bau

Haiming Jin

Hairui Yang

Haitao Qi

Haitao Yuan

Haitham M. Yousof

Hakan Başağaoğlu

Hakan Basargan

Hakan Sahin

Halil İbrahim Burgan

Hamadjam Abboubakar

Hamdy Mahmoud

Hamed Moayyed

Hamid Alinejad-Rokny

Hamid Baghani

Hamid Ghadiri

Hamid Mofidi

Hamid Reza Karimi

Hamid Saadatfar

Hamza Hanbali

Hamza Khan

Han Zhang

Hana M. Dobrovolny

Hana Tomášková

Hanan Hiba

Hang Li

Hang Ye

Hangchen $\mathrm{Yu}$

Hanno Hildmann

Hannu Laurila

Hans Georg Feichtinger

Hans-Peter Weikard

Hao Dong

Hao Yu

Hao Zhang

Haoxiang Yang

Haozhen Situ

Haozhi Pan

Hardik A. Marfatia

Harikumar Rajaguru

Harish Garg

Harith Kharrufa

Harleigh Christian Marsh

Harrie De Swart

Harry Haupt 
Harry Shaw

Harun Or-Roshid

Harun Pirim

Hasanen A. Hammad

Haseeb Athar

Hashem Bordbar

Hashim Habiballa

Hasmat Malik

Hassan Kamil

Hassan Kamil Jassim

Hassanen A. Hamad

Hassen Aydi

Hassène Gritli

Hattaf Khalid

Hayato Saigo

Hayden Julius

Hayder I. Mohammed

Hazem Al-Mofleh

He Yang

Héctor D. Menéndez

Héctor Migallón

Heinrich H. Nax

Helbert Eduardo Espitia

Hélder Lopes

Helena Campos

Helena Sit

Hélia Jacinto

Helmut Ahammer

Helmut Küchenhoff

Hemant Kumar Nashine

Hendrik Richter

Hendrik Van Maldeghem

Henrieta Hrablik Chovanova

Henry Gillow-Wiles

Henry Laniado

Henry-S. M. Chang

Herbert Lee

Herie Park

Hêriş Golpîra

Herminia I. Calvete Fernández

Heru Susanto

Hessam Golmohamadi

Heydar Toossian Shandiz

Heyong Wang

Hichem Eleuch

Hichem Mrabet

Hidetoshi Nakagawa

Hieu Pham

Higinio Ramos

Hijaz Ahmad

Hikmet Koyunbakan
Himanshu Nagpal

Himchan Jeong

Hirbod Assa

Hiroaki Yoshida

Hirofumi Izuhara

Hiroki Mizuochi

Hiroki Teragawa

Hiroshi Kuroda

Hiroyuki Kitahata

Hisao Ishibuchi

Hisashi Morioka

Ho Namgung

Hoang Pham

Homayun Motameni

Hong Gao

Hong Li

Hongfei Tang

HongGuang Sun

Hong-Jen Lin

Hong-Kun $\mathrm{Xu}$

Hong-Li Li

Hongliang $\mathrm{He}$

Hongshik Ahn

Hongwei Guo

Hong-Xuyen Thi Ho

Hongyu Liu

Hossein Aminikhah

Hossein Hassani

Houssem Bouchekara

Houssine Zine

Hovik Matevossian

Howard Levinson

Hristo Kiskinov

Hristo Manev

Hristo V. Kojouharov

Hsiuying Wang

Hsuan-Chu Chen

Huan Zhao

Huanfei Ma

Huanyu (Larry) Cheng

Huaping Chen

Hubert Anysz

Hubert Cecotti

Hudson Torrent

Hugo J. Bello

Hugo Valadares Siqueira

Hui Sun

Hui Zheng

Hui-Chin Tang

Huitian Lu

Hung-Jr Shiu 


Hung-Pin Huang
Hung-Yi Chuang
Huo Qunhai
Huong Thu Nguyen
Husam A. Foudeh
Husam Jasim Mohammed
Hüseyin Budak
Hüseyin Kamacı
Huseyin Kusetogullari
Hussain Mahdi
Hussein Rappel
Hwajeong Seo
Hwajoon Kim
Hyun Soo Chung
Hyung Kim
Hyunjo Jeong
I. Made Arnawa
I. Naci Cangul
Ian Jones
Ibrahim A. Abbas
Ibrahim M. Hezam
Ibrahim M. Sayed
Ibrahim Mohamed Diaaeldin
Ida Claudia Panetta
Idelfonso Nogueira
Idiano D'Adamo
Idris Ahmed
Ieva Meidutė-Kavaliauskienė
Ieva Misiunaite
Ignacio Javier Pérez
Ignacio Pérez-Rey
Ignazio Licata
Igor Andrianov
Igor Gruić
Igor Kozhukhov
Igor Mandel
Igor Mandric
Igor Nikolaev
Igor Rodionov
Igor V. Shevchuk
Igor Vujović
Igor Ya Subbotin
Igor Yadikin
Ihsan Jabbar Hasan
Ihsan Mohd Yassin
Ilaria Ferrando
Ildar Badretdinov
Ildefonso Castro-Infantes
Ildikó Kemény
ILEA Veronica
Ilia S. Kashchenko
Ho

Hung-Pin Huang

Ilias Elmouki

Ilias Pappas

Ilias S. Kotsireas

Ilona Södervik

Ilya A. Chernov

Ilya Boykov

Ilya G. Kovalenko

Ilya Levin

Ilya Smirnov

Imlak Shaikh

Immacolata Oliva

Imran Aziz

Imre Ferenc Barna

Imtiaz Ahmad

Imtiaz Mohammad Sifat

Indika Kumara

Indranil Ghosh

Indranil SenGupta

Indrasis Chakraborty

Inessa Matveeva

Inez Zwetsloot

Iñigo Sarría

Inmaculada Barranco-Chamorro

Ioan Batrancea

Ioan Bogdan Robu

Ioan Cristian Chifu

Ioan Tomescu

Ioana Opris

Ioannis D. Moscholios

Ioannis Dassios

Ioannis E. Antoniou

Ioannis E. Livieris

Ioannis E. Sarris

Ioannis Emmanouil

Ioannis K. Argyros

Ioannis Kyriakou

Ioannis Lamprou

Ioannis Mallidis

Ioannis P. Gravas

Ioannis Paraskevopoulos

Ioannis Th. Famelis

Ioannis Triantafyllou

Ioan-Viorel Banu

Ion Iancu

Ion Mihai

Ionel Bondoc

Ionel Tevy

Ionica Oncioiu

Ionut Brandusoiu

Ionut Daniel Geonea

Ionut Emil Iacob 


\author{
Ionut-Gabriel Farcas \\ Iosif Szeidert \\ Ira Valenzuela \\ Iren Valova \\ Irena Hinterleitner \\ Irena Jadlovska \\ Irene Crimaldi \\ Irene Díaz \\ Irene Ferrando Palomares \\ Irène Guessarian \\ Irene Nutini \\ Irene Oliveira \\ Irene Polo-Blanco \\ Ireneusz Kubiak \\ Ireneusz Miciuła \\ Ireneusz Winnicki \\ Irfan Ahmad Khan \\ Irfan Mustafa \\ Irina Albăstroiu \\ Irina Bashkirtseva \\ Irina Cristea \\ Irina E. Bocharova \\ Irina G. Burova \\ Irina Georgiana Mocanu \\ Irina Shevtsova \\ Irina Vinogradova-Zinkevič \\ Irvin Hentzel \\ Iryna Yaremchuk \\ Isa Abdullahi Baba \\ Isaac A. García \\ Isaac Harris \\ Isabel Vale \\ Isabela Roxana Birs \\ Isabella Torcicollo \\ Isaías González Pérez \\ Ishan Bajaj \\ Isidoros Perikos \\ İsmail Naci Cangül \\ Ismail Nikoufar \\ Ismini Lourentzou \\ Israel García-Alonso \\ Israel Pala Rosas \\ István Faragó \\ István Harmati \\ Itzamá López Yáñez \\ Iulia Hirica \\ Iulia-Alina Anton \\ Iuliana Botha \\ Iustina Alina Boitan \\ Iva Dokuzova \\ Ivan Area
}

Iván Blanco Chacón

Ivan Chipchakov

Ivan Cornejo

Ivan Ganchev Ivanov

Ivan Giannoccaro

Ivan Giorgio

Ivan Halkijevic

Ivan Izonin

Ivan Lukačević

Ivan Magdalenić

Ivan Pires

Ivan Šimeček

Ivan Sukin

Ivan Zaryadov

Ivana Budinska

Ivana Filipan

Ivar E. Vermeulen

Ivo Bukovsky

Ivona Brajević

Ivvan Valdez-Peña

Iwa Kuchciak

Iwona Bąk

Iwona Grobelna

Iwona Paprocka

Iwona Skrodzka

Iwona Włoch

Izabela Dembińska

Izabela Luiza Pop

Izabela Rejer

Izabela Świetlicka

Izabela Sztangret

Izhar Uddin

J. B. Heaton

J. Christian Attiogbé

J. Gaxiola-Camacho

J. H. Cho

J. J. Sylvia IV

J. Jumadi

J. Leonel Rocha

J. Thriveni

Jaan Janno

Jabar H. Yousif

Jacek Binda

Jacek Bogucki

Jacek Dziok

Jacek Gulgowski

Jacek Izydorczyk

Jacek Maciej Stankiewicz

Jacek Ryczyński

Jacob Skauvold

Jae Hoon Choi 


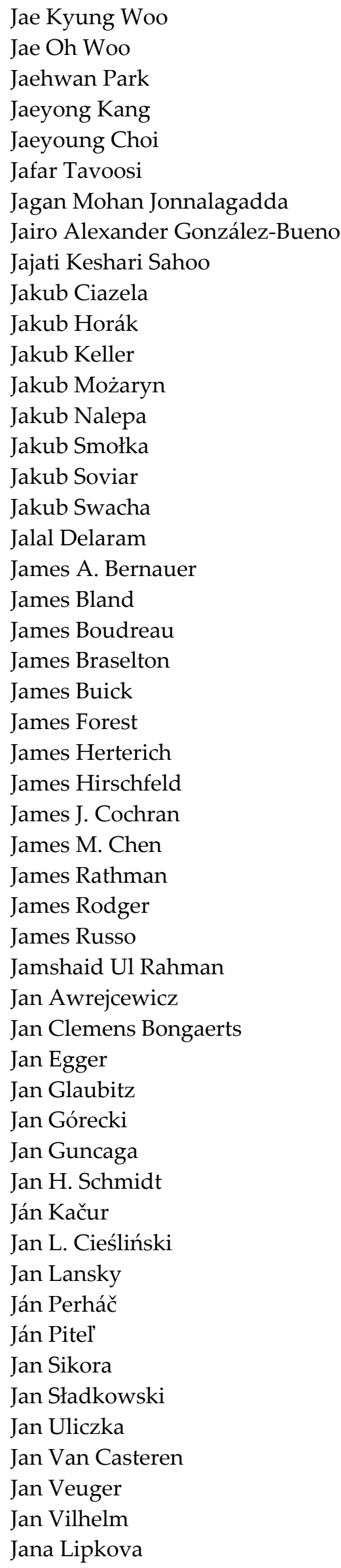

Jana Majerova

Jana Siebert

Janak Raj Sharma

Jane Ruseski

Janetta Culita

Janez Žerovnik

Janika Leoste

Janka Medová

Janos Abonyi

Janos Botzheim

János Sztrik

Janusz Brzdek

Janusz Dudczyk

Janusz Kołodziej

Janusz Rajda

Janusz Sosnowski

Jaouad Bennouna

Jarek Miszczak

Jari Hämäläinen

Jarmila Novotna

Jarmila Šebestová

Jaromír Vrbka

Jaron Sanders

Jaroslav Beránek

Jaroslav Hrdina

Jaroslav Vrchota

Jaroslaw Galkiewicz

Jarosław Rudy

Jarosław Wątróbski

Jaroslaw Ziolkowski

Jasdev Bhatti

Jasminka Alijagić

Jasna Prester

Jason Bramburger

Jason Lin

Jatinderkumar R. Saini

Javier Amezcua

Javier Borge-Holthoefer

Javier Cuenca

Javier De Lucas

Javier De Lucas Araujo

Javier E. Contreras-Reyes

Javier Fernandez

Javier Martínez Torres

Javier Oliver Muncharaz

Jawad Kazmi

Jaya Bishwal

Jaya Wen

Jean C. Perez

Jean Vaillant

Jean-Charles Pinoli 


\begin{tabular}{|c|c|}
\hline Jean-François Angers & Jianjun (Paul) Tian \\
\hline Jean-Marie Maillard & Jian-Qiang Wang \\
\hline Jean-Michel Tucny & Jianxin Jia \\
\hline Jean-Paul Fischer & Jianzhong Wang \\
\hline Jean-Philippe Aguilar & Jiaqi Jiang \\
\hline Jean-Pierre Gazeau & Jiawei Li \\
\hline Jean-Pierre I. Van Der Rest & Jiaxin Zhang \\
\hline Jean-Pierre Magnot & Jiayao Chen \\
\hline Jeff Camm & Jichun Li \\
\hline Jeffrey Buckley & Jie Liu \\
\hline Jelena Kilić Pamuković & Jie Meng \\
\hline Jelena Mušanović & Jie Yang \\
\hline Jelena Šaković Jovanović & JIE Zhong \\
\hline Jelena Vujakovic & Jiejun $\mathrm{Hu}$ \\
\hline Jen-Chih Yao & Ji-Eun Lee \\
\hline Jen-Der Day & Ji-huan He \\
\hline Jenna O'Dell & Jill Faudree \\
\hline Jenni Redifer & Jim Coykendall \\
\hline Jennifer Darling-Aduana & Jim W. Kay \\
\hline Jenny Pagge & Jin Kyung Kwak \\
\hline Jens Jensen & Jin Su Jeong \\
\hline Jeong Hwan Lee & Jin Wang \\
\hline JeongGon Lee & Jin Zhou \\
\hline Jérôme Detemple & Jinfeng Liu \\
\hline Jérôme Mendes & Jingwei Song \\
\hline Jerzy Balicki & Jingwen Song \\
\hline Jerzy Baranowski & Jingxu Zheng \\
\hline Jerzy Kisilowski & Jinhua Mi \\
\hline Jerzy Weres & Jinku Park \\
\hline Jerzy Winczek & Jinlian Ren \\
\hline Jerzy Witold Wiśniewski & Jintai Ding \\
\hline Jessica Rodrigues & Jinze $\mathrm{Xu}$ \\
\hline Jesús López Belmonte & Jiří Ajgl \\
\hline Jesús Marín Solano & Jiri Cecrdle \\
\hline Jesús Montejo Gámez & Jiří Močkoř \\
\hline Jesús Rodríguez-López & Jiří Náprstek \\
\hline Jhony Alexander Villa-Ochoa & Jiri Petrzela \\
\hline Jia Ping Wu & Jiří Witzany \\
\hline Jiafa $\mathrm{Xu}$ & Jize Zhang \\
\hline Jiafu Su & Joan Carles Artes \\
\hline Jiahang Yuan & Joana Cruz \\
\hline Jiaming $\mathrm{Na}$ & Joanna Janicka \\
\hline Jian Gao & Joanna Kołodziejczyk \\
\hline Jianbin Qiu & Joanna Raczek \\
\hline Jianchao Cai & Joanna Weissenberg \\
\hline Jiandong Ren & Joanna Wyrwa \\
\hline Jianfei Huang & João Cabral \\
\hline Jianfeng Hou & João Carlos Ferreira \\
\hline Jianguo Liu & Joao Duraes \\
\hline Jianhua Wu & João Fialho \\
\hline Jianing Han & João Leitão \\
\hline
\end{tabular}




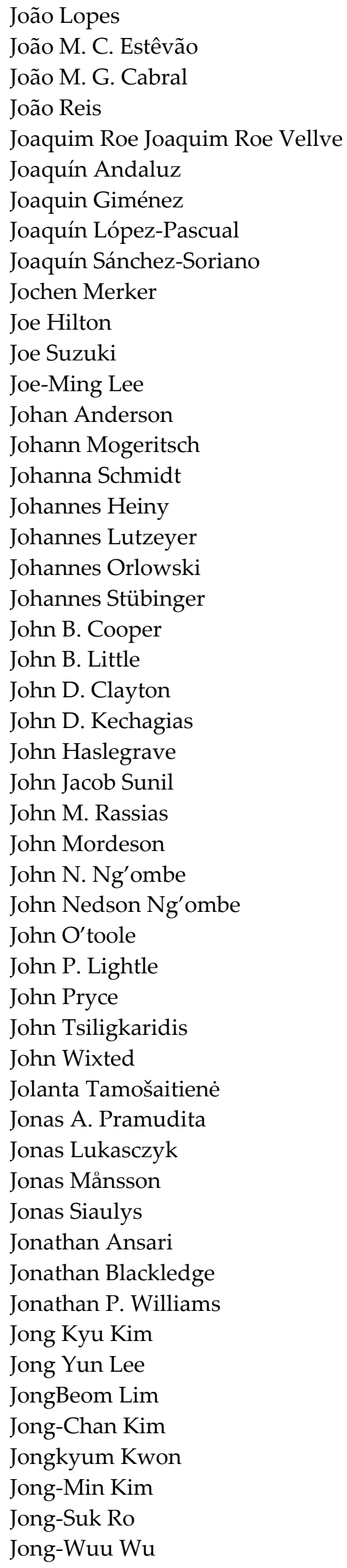

Jonnalagadda Srinivas

Jordan Kralev

Jordi Garcia

Jordi Gómez Castilla

Jordi Nin

Jordi Ripoll

Jordi-Roger Riba

Jörg Koppitz

Jorge Cerqueiro-Pequeño

Jorge De Andres Sanchez

Jorge Delgado Gracia

Jorge E. Macias-Diaz

Jorge Eliecer Hernandez Hernandez

Jorge Fatelo

Jorge Herrera-Tapia

Jorge Igual

Jorge Maestre Vidal

Jorge Muñoz Yañez-Barnuevo

Jorge O. Moreno

Jorge Oliveira

Jorge Rios

Jorge Suzuki

Jorma Joutsenlahti

José A. F. Costa

Jose A. Iranzo

José Alberto Rodrigues

José Alonso Solís-Lemus

José Álvarez-García

Jose Angel Aranda

José António Candeias Bonito Filipe José Antonio De Diego Onsurbe Jose Antonio Domínguez-Navarro Jose Antonio Fernández Gallardo José Antonio Filipe José Antonio Moraño Jose Antonio Roldán-Nofuentes José Antonio Sánchez Pelegrín Jose Cánovas José Carlos Alcantud Jose De Jesus Rubio Jose Domínguez Jose Fernandez José Francisco Gómez-Aguilar Jose Gaite José Gómez-Galán José Gregorio Gomez-garcia Jose Guivant José J. Alonso Del Rosario Jose J. López-Espín José L. López José Luis Felipe 


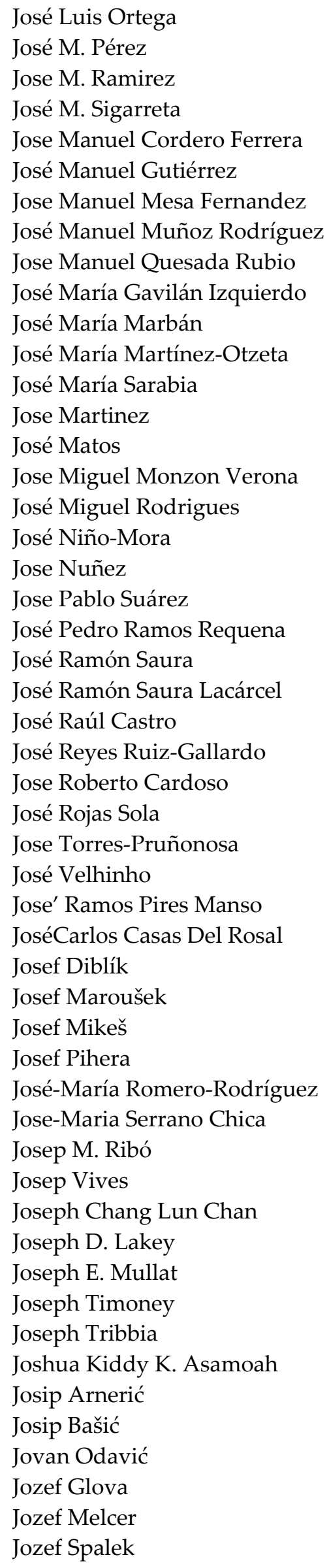

József Lennert

József Z. Farkas

Juan Antonio Morente-Molinera Juan Antonio Sicilia-Montalvo Juan Carlos Hernández-Gómez Juan Carlos Valenzuela-Tripodoro Juan De Dios Pérez Juan E. Nápoles Valdes Juan E. Trinidad Segovia Juan Eloy Ruiz-Castro Juan Enrique Martínez-Martínez Juan F. Mañas-Mañas Juan Francisco Sánchez-Pérez Juan Francisco Trigo Juan Frausto Juan G. Alcazar Juan Garcia Guirao Juan Ignacio García-García Juan J. García-Machado Juan J. Leiva Olivencia Juan José Fernández Juan Luis Piñeiro Juan Luis Rubio Juan M. Santos-Gago Juan Manuel Pérez-Pardo Juan Manuel Rodríguez Prieto Juan Ortega Juan P. Vasconez Juan Pedro Martínez Ramón Juan R. Torregrosa Juan Ramón García Rozas Juan Sebastian Angarita Zapata Juana-María Vivo Juarez Bento Da Silva Jude Hemanth Juergen Bierbrauer Jugal Mohapatra Jui-Yung Chang Julia Eisenberg Julia Haba-Osca Julia Jones Julia Maria Pavan Soler Julian A. D. Allagan Julian S. Evans Juliano Fagundes Goncalves Julie Vaiopoulou Julien Lesouple Julio A. Cergo Julio Becerra Guerrero Julio Zamora Jun Liu 


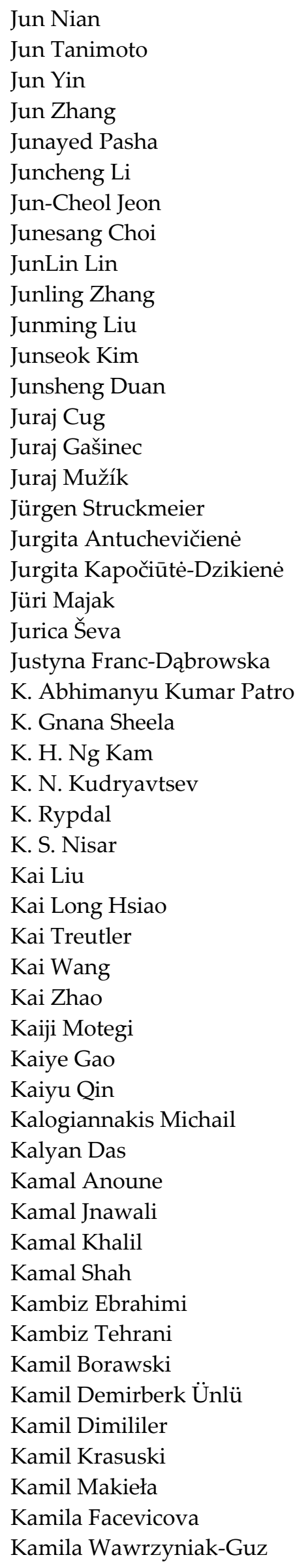

Kamsing Nonlaopon

Kanak Kalita

Kanel Alexey

Kang Liu

Kanit Mukdasai

Kao-Yi Shen

Kapil Juneja

Karel Janda

Karel Macků

Karim Aliakbari

Karina Joyce Wilkie

Karl Andersson

Karl Svozil

Karl-Joachim Wirths

Karol Gryszka

Karthikeyan Rajagopal

Karuppusamy Loganathan

Karyn A. Allee-Herndon

Kasamsuk Ungchittrakool

Katarína Čunderlíková

Katarína Hriňáková

Katarina Kostelić

Katarina Rogulj

Katarina Valaskova

Katarina Zvarikova

Katarzyna Bialas

Katarzyna Boratyńska

Katarzyna Smędzik-Ambroży

Katarzyna Trabka-Wieclaw

Kateřina Jančaříková

Katerina Kabassi

Katerina Polyakova

Katerine Romeo

Kateryna Vlasenko

Katharina Kusejko

Kathleen Nolan

Kathryn Daltorio

Kathryn Y. Manning

Katina Kralevska

Kauko Leiviskä

Kaushik Jana

Kavita Goyal

Kay Owens

Kazuharu Bamba

Kazuhiro Ogata

Kazuki Ikeda

$\mathrm{Ke} \mathrm{Bi}$

Ke Feng

Ke Shen

Ke Shi

Ke Wang 
Kehui Sun

Kei Hirose

Kei Nakagawa

Keiko Uohashi

Keisuke Teramoto

Keith Andrew

$\mathrm{Ke}$-Lin Du

Kelmara Mendes Vieira

Kenichi Kasamatsu

Kenjiro Miura

Kenneth C. Land

Kenneth Y. T. Lim

Kershen Huang

Keshav Singh

Kesra Nermend

Kevin Burrage

Ke-Vin Chang

Kevin Knudson

Kevin Zelaya

Ke-Xue Li

Khairy Sayed

Khaled Alawasa

Khaled Gepreel

Khaled Giasin

Khaled Saad

Khalid Khan

Khalid Rashid

Khalide Jbilou

Khanh Chau Le

Khosro Khandani

Khurram Hameed

Khursheed J. Ansari

Ki Jung Yoon

Kia Dashtipour

Kiattipoom Kiatkawsin

KiChun Lee

Kifayat Ullah

Kim Meow Liew

Kin Hing Lo

Kirill Krinkin

Kirill Kuzmin

Kirill Pervakov

Kirti Yenkie

Kiseob Lee

Kishor Datta Gupta

Kitti Páleníková

Kıvanç Tatar

Ki-Yeol Shin

Kjetil Uhlen

Klaudia Smoląg

Klaus Ackermann
Klaus Morawetz

Klaus Wohlrabe

Koji Yokote

Konrad Furmańczyk

Konstantin Alexandrovich Rybakov

Konstantin Burmistrov

Konstantin Isupov

Konstantin Kozlov

Konstantin Kuck

Konstantin Nikolic

Konstantin Samouylov

Konstantin Sharov

Konstantin Zhukovsky

Konstantinos A. Lazopoulos

Konstantinos Demertzis

Konstantinos Giannoutakis

Konstantinos Gkillas

Konstantinos Kokkinos

Konstantinos Kotis

Konstantinos Limniotis

Konstantinos Prokopidis

Konstantinos Zografos

Korawit Orkphol

Koriath Hans-Joachim

Kostaq Hila

Kostas Vergidis

Kotb Tawfiq

Kovács László

Ko-Wei Huang

Krasimira Borislavova Ivanova

Krassimir Atanassov

Krassimir Georgiev

Krassimira Vlachkova

Kris Van Der Zee

Krishanu Roy

Kristina Šutienė

Krystian Jobczyk

Krzysztof A. Cyran

Krzysztof Chwastek

Krzysztof Dmytrów

Krzysztof Domino

Krzysztof Foit

Krzysztof Gdawiec

Krzysztof Gornicki

Krzysztof Grygierek

Krzysztof Kania

Krzysztof Klimaszewski

Krzysztof Kluza

Krzysztof Kukielka

Krzysztof Piasecki

Krzysztof Psiuk-Maksymowicz 
Krzysztof Puszynski

Krzysztof Rogowski

Krzysztof Sibilski

Krzysztof Stopka

Krzysztof Szajowski

Krzysztof Tomczyk

Krzysztof Tomiczek

Krzysztof Trojanowski

Krzysztof Wałęsa

Krzysztof Waliszewski

Krzysztof Wołk

Ksenija Smoljak Kalamir

Kuang-Hsun Shih

Kuei-Hu Chang

Kuen-Suan Chen

Kui Zhang

Kulina Hristina

Kumar Shivam

Kun Li

Kung-jui Pai

Kunichika Tsumoto

Kun-Li Wen

Kunwar Virendra Singh Chouhan

Kuo-Ping Chiao

Kurbatov Vitalii

Kurubaran Ganasegeran

Kutubuddin Ansari

Kwang Sik Kim

Kwang-Hoon Kim

Kwangsup Shin

Kyaw Hein

Kyeongjun Lee

Kylie Swanson

Kyriaki Kostoglou

Kyriaki-Nefeli D. Malamaki

Kyriakos Flouris

Kyriakos Papadopoulos

Kyung-Yong Chung

L. F. Spevak

Laddu Bhagya Jayasinghe

Lahari Sengupta

Laith Abualigah

Lakshmanan Shanmugam

Lanre Akinyemi

Lara Orcos

Larisa Beilina

Larissa Batrancea

Larry Glasser

Lars Holzäpfel

Laszlo Barna Iantovics

László Berényi
Laszlo Csirmaz

László Kovács

László Németh

László Pokorádi

László Sipos

László Vasa

Lato Pezo

Laura Bravi

Laura Delgado

Laura Delgado-Martín

Laura Diosan

Laura Gardini

Laura Lea Sacerdote

Laura Paladino

Laura Pezza

Laura Screpanti

Laura-Maria Dogariu

Laurent Donzé

Laurent Lemarchand

Laurentiu Mihai Ionescu

Lauri Viitasaari

Laurian-Ioan Piscoran

Laurie D. Edwards

Lawrence Smolinsky

Lazaros Moysis

Lazhar Homri

Leandro Magalhães

Leandro Marin Muñoz

Leandro Tavares

Lech Górniewicz

Lechang Yang

Leda D. Minkova

Lefteris Doitsidis

Lei Wang

Leila Marek-Crnjac

Lelys Bravo

Lena Mihova

Lenka Lhotska

Lenka Mihoković

Leo H. Chan

Leo Tiokhin

Leonardo Colombo

Leonardo Machado Da Rosa

Leonardo N. Ferreira

Leonardo Primavera

Leoneed Kirilov

Leoneed M. Kirilov

Leonel Jorge Ribeiro Nunes

Leonel Nunes

Leonid Hanin

Leonid Kritskov 
Leonid P. Pryadko

Leonid Shaikhet

Leonid V. Bogdanov

Leonie Hallo

Leopoldo Eduardo Cárdenas-Barrón

Leopoldo Greco

Lester Ingber

Leszek Chomacki

Leszek Gasinski

Leszek Marcinkowski

Leung Lung Chan

Lev B. Klebanov

Lev Eppelbaum

Lev Kazakovtsev

Lev Ryashko

Levente Czumbil

Lia E. Aciu

Liana Claudia Salanță

Liang Chu

Liang Liang

Liang Yan

Liang-Bi Chen

Lianggui Liu

Liangliang Cheng

Liangliang Xiao

Lianyu Yu

Libor Pekař

Lida J. Uribe-Flórez

Lieu-Hen Chen

Lihai Zhang

Lihua Li

Liliana Guran

Lilla Koreňová

Lin $\mathrm{Li}$

Lin Wang

Lina Higueras-Rodriguez

Lina Novickyte

Linas Petkevičius

Linfei Yin

Ling Wei

Lingfa Lu

Lingqiang $\mathrm{Li}$

Lingwei $\mathrm{Xu}$

Lingxiao Wang

Lining Jiang

Lionel Pichon

Liping Liu

Liu Wei

Liudmila Ya Banakh

Liviu Iulian Palade

Liviu Popescu
Liviu-Adrian Cotfas

Liwen Chen

Lixin Shen

Ljiljana Primorac Gajčić

Ljubica Kazi

Llanos Tobarra

Loi Lei Lai

Loïc Brevault

Long Jin

Lorand Szabo

Loredana Bellantuono

Loredana Ciurdariu

Lorentz Jäntschi

Lorenz Schwachhöfer

Lorenzo A. Sadun

Lorenzo Cerboni Baiardi

Lorenzo Escot

Lorenzo Fiorineschi

Lorenzo Luperi Baglini

Lorenzo Putzu

Lorenzo Ruffoni

Lorenzo Trapani

Lourdes Tello

Lubin G. Vulkov

L'ubomír Antoni

Lubos Buzna

Luboš Smolík

Luc Vrancken

Luca Andrea Ludovico

Luca Chiantini

Luca D'Acierno

Luca Dede'

Luca Di Persio

Luca Gemignani

Luca Lista

Luca Pancioni

Luca Pegolotti

Luca Rossini

Luca Sandrini

Lucas Martínez-Bernabéu

Lu-Chuan Ceng

Lucia Bandiera

Lucía Inglada-Pérez

Lucia Lopez Somoza

Lucia Ratiu

Lucia Rocchi

Lucia Svabova

Lucian Lupu-Dima

Lucian Trifina

Lucija Brezočnik

Lucio Centrone 
Lucio Palazzo

Lucjan Sapa

Ludivine Martin

Ludovic Goudenège

Ludovic Montastruc

Luigi Aldieri

Luigi C. Berselli

Luigi Fortuna

Luigi Foschini

Luigi Lentini

Luigi Marangio

Luigi Rarità

Luis Carlos Contreras

Luis Coelho

Luis Enrique Moreno Lorente

Luis Filipe Almeida Bernardo

Luís Filipe Ribeiro

Luís Frazão

Luis García Barrachina

Luis Gómez Déniz

Luis Hernández-Callejo

Luis J. Rodríguez-Muñiz

Luis Manuel Sánchez Ruiz

Luis Margalho

Luis Miguel Grilo

Luis P. Castro

Luis Pérez Rojas

Luis Pérez-Domínguez

Luis Sanchez

Luis Santos

Luis Seco

Luisa Fattorusso

Luis-Alberto Casado-Aranda

Luiz C. B. Da-Silva

Luiz Guerreiro Lopes

Luiz Roberto Evangelista

Luka Grubisic

Luka Rumora

Lukas Burgholzer

Lukáš Kučera

Lukasz Bohdal

Łukasz Chomątek

Łukasz Drobiec

Lukasz Jelen

Łukasz Knypiński

Łukasz Sajewski

Łukasz Smaga

Łukasz Sobaszek

Łukasz Tomczyk

Łukasz Wieczorek

Łukasz Żyłka
Luminița Ionescu

Lung-Hui Chen

Lutful Karim

Lydia Dux-Santoy Hurtado

Lyubka Aleksieva

Lyubov Khomyakova

Lyudmila Bel'skaya

Lyudmila Kiryanova

M. A. Korolev

M. Ali Ülkü

M. Angeles Martínez

M. Arulperumjothi

M. Asunción Lubiano

M. Glòria Barberà-Mariné

M. J. Huntul

M. K. Marichelvam

M. L. Puertas

M. M. Bhatti

M. Muthtamilselvan

M. Nishat Akhtar

M. P. Chaudhary

M. Pilar Vélez

M. Premkumar

M. R. Hematiyan

M. R. Safaei

M. S. Osman

M. S. Zobaer

M. Saqib

M. Shamim Kaiser

M. Syed Ali

M. Teresa Ortuño

M. V. A. Raju Bahubalendruni

M. Zakarya

Maan A. Rasheed

Maanak Gupta

Maarten Punt

Macarena Castellary López

Maciej Błaszak

Maciej Majewski

Maciej Niedostatkiewicz

Maciej Przanowski

Maciej Przybyłek

Maciej Romaniuk

Maddalena Marchelli

Maddalena Nonato

Madeleine Dobson

Madeline Al Tahan

Madhuri S. Mulekar

Magda Dettlaff

Magdalena Matuszak

Magdalena Mrokowska 
Magdalena Osińska

Magdalena Szymczyk

Mahadi Ddamulira

Mahdi Imani

Mahdi Khosravy

Mahendra Kumar Gupta

Maher Maalouf

Mahmod Othman

Mahmood Alkhassaweneh

Mahmoud Abdel-Aty

Mahmoud Afshari

Mahmoud Bayat

Mahmoud El-Borai

Mahmoud El-Morshedy

Mahmoud Golabi

Mahmoud Hasan Alrefaei

Mahmoud Ibrahim

Mai The Vu

Maja Andric

Maja Mesko

Majeed Abdul

Majid Mazouchi

Majid Tavasoli

Majorkowska-Mech Dorota

Mäkipää Toni

Makrina Agaoglou

Maksim Dolgopolik

Maksim Kukushkin

Malcolm Brady

Małgorzata Gawlik-Kobylińska

Małgorzata Grzelak

Małgorzata Iwanek

Malgorzata Jasiulewicz-Kaczmarek

Małgorzata Łatuszyńska

Malgorzata Pankowska

Małgorzata Pawłowska

Małgorzata Węgrzyńska

Malik Zaka Ullah

Malte Gerhold

Man Ho Ling

Mangey Ram

Manh-Duc Nguyen

Man-Hoe Kim

Manik Sharma

Manish Kumar

Manjari Gupta

Manoj Kumar

Manotosh Kumbhakar

Manuel Adrián Acuña-Zegarra

Manuel Arana-Jimenez

Manuel De La Sen
Manuel De Leòn

Manuel Ferreira

Manuel Florindo Alves Meirinhos

Manuel Lafond

Manuel Ojeda-Aciego

Manuel Prado-Velasco

Manuel Ruiz Galán

Manuel Streicher

Manuela Ingaldi

Manuela Tvaronavičienè

Manzoor Hussain

Maosheng Xiong

Mao-Ting Chien

Mar Arenas-Parra

Marat Mukhametzhanov

Marat V. Yuldashev

Marc Dupuis

Marc Jacquinet

Marcel Antal

Marcel Ausloos

Marcel Bogdan

Marcel Clerc

Marcel Walter

Marcela Popescu

Marcelina Mocanu

Marcella Nicolini

Marcellin Atemkeng

Marcello Delitala

Marcello Fera

Marcelo Kaminski Lenzi

Marcin Fałdziński

Marcin Gajewski

Marcin Hernes

Marcin J. Schroeder

Marcin Jesionek

Marcin Kaminski

Marcin Klimek

Marcin Lawnik

Marcin Łoś

Marcin Makowski

Marcin Nowak

Marcin Sosnowski

Marcin Wątorek

Marcin Wesołowski

Marcin Ziółkowski

Marcin Zych

Márcio A.F. Martins

Marco A. Palomino

Marco Antonio Marquez-Vera

Marco Berardi

Marco Bindi 
Marco Bruno Luigi Rocchi

Marco Danilo Claudio Torri

Marco Domaneschi

Marco Jose

Marco Lombardi

Marco Martins Afonso

Marco Papi

Marco Pedroni

Marco Riani

Marco Trombetti

Marco Viola

Marcoen J. T. F. Cabbolet

Marcos Cavenaghi

Marcos Raydan

Marcos Tostado-Véliz

Marek Angowski

Marek Bauer

Marek Błasik

Marek Brabec

Marek Galewski

Marek Golasinski

Marek J. Druzdzel

Marek Krok

Marek Lampart

Marek Landowski

Marek Lefik

Marek Rydel

Marek Sawerwain

Marek Skarupski

Marek Sylwester Tatara

Marek Urban

Marek Zborowski

Margaret Marshman

Margareta Gardijan Kedžo

María Alonso-García

Maria Aluchna

María Álvarez Hernández

Maria Andronic

Maria Angeles Garcia-Ferrero

Maria Bostenaru

Maria Bostenaru Dan

Maria Bruzon

María C. Cañadas

María Carmen Carnero

Maria Cieśla

Maria Cristina Costa

Maria Datcheva

María De La Cruz Del Río-Rama

Maria Del Carmen Ramos-Herrera

María Del Carmen Valls Martínez

María Del Consuelo Ruiz Rodríguez
María Del Mar Fernández-Martínez

Maria Do Castelo Gouveia

Maria Do Rosário Grossinho

Maria Dobritoiu

María Dolores Gadea Rivas

Maria Eduarda Ferreira

Maria Esmeralda Gonçalves

María Estrella Sousa Vieira

Maria Farcaseanu

Maria Flavia Mammana

Maria Francesca Milazzo

Maria Gagarina

Maria Gratiela Ianos

Maria Grazia Russo

Maria Isabel Berenguer

María Isabel Lamas

María Isabel Parra

María Jesús Delgado Rodríguez

María Jesús Segovia Vargas

Maria João Valente Da Silva Couto

María José Cáceres

Maria Jose Casany

Maria Jose Gomez-Silva

Maria José Lumini Landeiro

Maria Korovina

Maria Krechowicz

Mária Kúdelčíková

Maria L. Manca

Maria Laura Manca

Maria Lopez-Herrero

Maria Lucia Sampoli

Maria Luisa Villani

Maria Luminita Scutaru

Maria M. Nascimento

Maria Mansanet-Bataller

Maria Mariani

Maria Matusiewicz

María Morales

Maria Mrówczyńska

María Napal

María Navascues

Maria P. Beccar-Varela

Maria Padrós Cuxart

Maria Papadopoulou

Maria Pilgun

Maria Rosaria Guarini

Mária Slavíčková

Maria Speciale

María Teresa Sánchez

Maria Vasileva

Mária Ždímalová 
María-Luisa Pérez-Delgado

Marian Dragoi

Marian Ioan Munteanu

Marian Kachniarz

Marian Pompiliu Cristescu

Marian Wysocki

Mariana Beňová

Mariana Marčoková

Marianito Rodrigo

Mariano Alarcón

Mariano Amo-Salas

Mariano Torrisi

Marianthi Batsila

Marie Christine Firpo

Marie Luisa Schaper

Marie Paz E. Morales

Marie Perrot

Marie-Anne Guerry

Marietta Kiss

Mariia A. Molodchik

Marija Maksimović

Marija S. Najdanović

Marilena Crupi

Marilena Pannone

Marin Marin

Marina Alexandra Pedro Andrade

Marina Murillo-Arcila

Marina Paola Banchetti-Robino

Marina Resta

Marina Vasiljeva

Marina Yashina

Mario Abundo

Mario Alberto Martínez Núñez

Mario Bukal

Mario De Salvo

Mario Gionfriddo

Mario Grande-de-Prado

Mario Maggi

Mario Martínez Pizarro

Mário Nuno Mata

Mario Versaci

Marisa Faggini

Marius Gavrilescu

Marius Iulian Mihailescu

Marius Mihai Birou

Marius Pislaru

Marius Popescu

Marius Tucsnak

Mariusz Buciakowski

Mariusz Ciesielski

Mariusz Oszust
Mariusz Pleszczyński

Mariusz Salwin

Mariya Konsulova-Bakalova

Marjan Golob

Marjan Mernik

Marjan Milenkov

Mark D. Roberts

Mark Harris

Mark Kelbert

Mark Sh. Levin

Mark Sussman

Mark Wohar

Marko Delimar

Marko Gulić

Marko Kostić

Marko Maliković

Marko Merdzan

Markos G. Tsipouras

Markus Hainy

Markus Hertrich

Markus Lazar

Markus Puchinger

Marta Bistron

Marta Dziechciarz

Marta Harničárová

Marta Molina Huelva

Marta Reboiro

Marta Skiba

Marta Susana Ribeiro Ferreira

Martha L. Abell

Martin Baca

Martin Beer

Martin Biewen

Martin Bohata

Martin Čech

Martin Cenek

Martin Daumiller

Martin Eling

Martin Folta

Martin Glanzer

Martín H. Cruz-Rosales

Martin Hecht

Martin Hell

Martin Ibl

Martin Kalina

Martin Kenyeres

Martin Kotyrba

Martin Lanser

Martin Lněnička

Martin Straka

Martin Valtierra-Rodriguez 


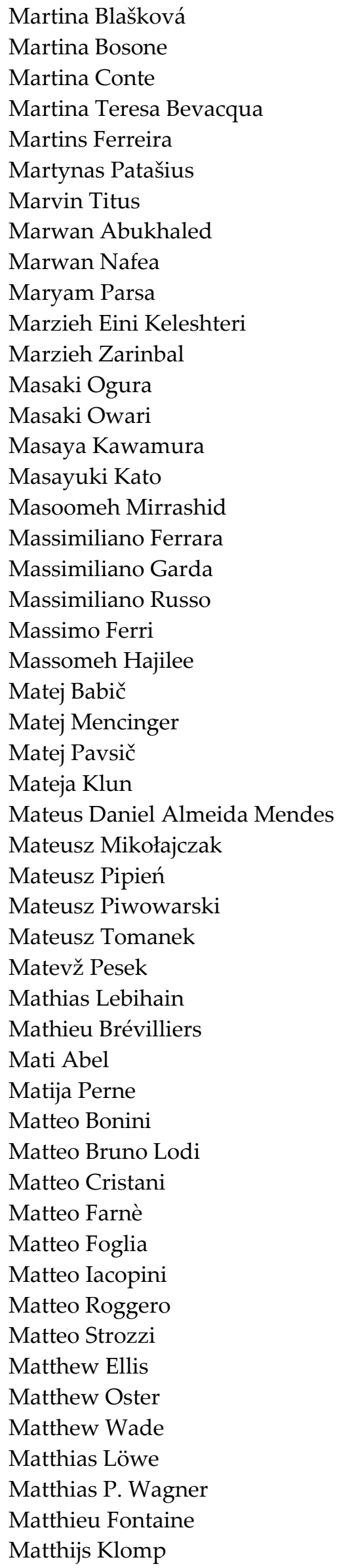

Martina Blašková

Mattia Fogagnolo

Matúš Šucha

Maurizio Ceseri

Maurizio Tavelli

Mauro Gaspari

Mauro Mezzini

Mauro Passacantando

Max Menzies

Max Winkler

Maxim A. Shishlenin

Maxim Deryabin

Maxim Dulebenets

Maya Stoyanova

Maziar Yazdani

Mazin Abed Mohammed

Mazyar Ghadiri Nejad

Md Abdul Quddus

Md Al Masum Bhuiyan

Md Ashraful Alam

Md Junayed Hasan

Md Mominul Ahsan

Md Sazzad Hosen

Md Shafiullah

Md Wahiduzzaman

Md. Irfanul Haque Siddiqui

Md. Shakhaoath Khan

Mee Seong Im

Megan Mihail

Mehdi Asadi

Mehdi Foumani

Mehdi Hosseinzadeh

Mehdi Khojastehpour

Mehdi Nadjafikhah

Mehdi Neshat

Mehdi Rahmati

Mehdi Salimi

Mehdi Shadab Far

Mehdi Teimouri

Mehmet Aydin

Mehmet Dorduncu

Mehmet Senel

Mehmet Yavuz

Mehran Kiani-Oshtorjani

Meisam Ranjbari

Meksianis Ndii

Meleshko Meleshko Sergey

Melike Aydoğan

Mélissa Rossi

Meng Leong How

MengChu Zhou

Meng-Leong How 


\author{
Mercedes Claramunt \\ Mercedes Hidalgo-Herrero \\ Mercedes Rodríguez-Sánchez \\ Mercedes Ruiz \\ Merica Slišković \\ Merten Stender \\ Merve Kester Thomas \\ Miao Ling Wang \\ Mica S. Stankovic \\ Michael (Michail) Th. Rassia \\ Michael A. Kouritzin \\ Michael Barton \\ Michael Berman \\ Michael Connell \\ Michael D. Spartalis \\ Michael D. Steele \\ Michael Evans \\ Michael F. Shlesinger \\ Michael Grabchak \\ Michael Guckert \\ Michael Heinrich Baumann \\ Michael Höft \\ Michael Jankowski \\ Michael Kearney \\ Michael Lata \\ Michael Lydeamore \\ Michael Mangold \\ Michael Pokojovy \\ Michael Radin \\ Michael Sadovsky \\ Michael Schlosser \\ Michael Th. Rassias \\ Michael Thomas Wolfinger \\ Michael Vannier \\ Michael Voskoglou \\ Michael Wester \\ Michael Weyrauch \\ Michaela Bednárová \\ Michał Awtoniuk \\ Michał Bartyś \\ Michal Berkowitz \\ Michal Bizzarri \\ Michal Buszko \\ Michał Gostkowski \\ Michal Hnatic \\ Michal Jasinski \\ Michal Kelemen \\ Michał Klichowski \\ Michal Kvet \\ Michał Mosiałek \\ Michal Niezabitowsky
}

Michal Pesta

Michal Pešta

Michał Popławski

Michal Puskar

Michal Staš

Michał Stasiak

Michał Urbaniak

Michał Wasik

Michał Witold Przewoźniczek

Michal Zamboj

Michalina Błażkiewicz

Michel Nguiffo Boyom

Michel Planat

Michel Riguidel

Michel Rouleux

Michele Ambrosanio

Michele Calì

Michele Rocca

Michelle Carey

Michelle Stephan

Mieczysław Cichoń

Miglena Koleva

Migue Angel Negrin

Miguel Ángel García-March

Miguel Ángel López Guerrero

Miguel Ángel Montes

Miguel Casero

Miguel Garcia Torres

Miguel Lorenzo

Miguel Lozano

Miguel Martínez García

Miguel Pineda

Mihaela Codruta Ancuti

Mihaela Colhon

Mihaela Hnatiuc

Mihaela Neamtu

Mihaela Simionescu

Mihaela-Diana Oancea-Negescu

Mihai Andries

Mihai Carp

Mihai Ciuc

Mihai Daniel Roman

Mihai Gavrilas

Mihai Mihailescu

Mihail Busu

Mihail Konstantinov

Mike Oluwatayo Ojo

Mike Tsionas

Mikhail A. Artemov

Mikhail Altaisky

Mikhail Babenko 
Mikhail Basarab

Mikhail Bragin

Mikhail Fistul

Mikhail Kalmykov

Mikhail Kolev

Mikhail Oet

Mikhail P. Galanin

Mikhail P. Gryaznevich

Mikhail Sheremet

Mikhail Stolbov

Mikhail V. Golub

Miklos Hoffmann

Miklos Ronto

Mikyung Shin

Milan Hladik

Milan Lj. Zlatanović

Milan Pokorný

Milena Petkova

Milena Wrobel

Milica Andjelic

Milos Birtus

Milos Milanovic

Milos Seda

Miloslav Vlasak

Miloslav Znojil

Milton Ferreira

Milton Rosa

Miluše Vitečková

Mimica R. Milošević

Min Lun Wu

Min Son

Min Wang

Min Wu

Min Xiong

Min Zhang

Minakhi Rout

Ming Li

Ming Liu

Ming Yang

Mingchang Chih

Mingchu Li

Mingda Zhou

Ming-Feng Ge

Ming-Fu Hsu

Mingji Zhang

Ming-Lang Tseng

Mingmian Cheng

Mingyang Lu

Minh-Chien Trinh

Min-Jie Luo

Minkyoung Kim
Minzhang Zheng

Miodrag Lovric

Miodrag Spalević

Miqing Li

Mir Sajjad Hashemi

Mircea Cimpoeas

Mircea Crasmareanu

Mircea Dulău

Mircea Gabriela

Mircea Hulea

Mircea Merca

Mircea Neagoe

Mircea-Bogdan Radac

Mirco Sturari

Mirela Catalina

Mirela Dragan

Miriam Vignando

Mirjana Pavlović

Mirjana Pejic Bach

Mirna Džamonja

Miron Zapciu

Miroslav Drljaca

Miroslav Halilovič

Miroslav Hristov

Miroslav Kelemen

Miroslav Kureš

Miroslav Langer

Miroslav Spacek

Miroslava Antić

Miroslava Nedyalkova

Miroslava Růžičková

Miroslaw Kordos

Mirosław Krzyśko

Mirosław Kurkowski

Mirpouya Mirmozaffari

Mirzosaid Sultonov

Mislav Trbušić

Mitali Sarkar

Mitianiec Wladyslaw

Mitica Craus

Mitică Craus

Mittal Mohit

Mladen Zrinjski

Mobayode Akinsolu

Modesar Shakoor

Modhurima Dey Amin

Modjtaba Ghorbani

Mogens Steffensen

Mohamad Aslani

Mohamed Abdel-Nasser

Mohamed Abdelsabour Fahmy 
Mohamed Ahmed Moustafa Hassan

Mohamed Amine Ben Farah

Mohamed Amine Ferrag

Mohamed Amouch

Mohamed Ben Haj Frej

Mohamed CH-Chaoui

Mohamed Darwish

Mohamed Gobashy

Mohamed Hamdaoui

Mohamed Houas

Mohamed Ibrahim

Mohamed Ibrahim Abbas

Mohamed Jleli

Mohamed K. El-Nemr

Mohamed Lotfi

Mohamed M. Mousa

Mohamed R. Abonazel

Mohamed Salahudeen

Mohammad Amin Hariri-Ardebili

Mohammad Asim

Mohammad Aslefallah

Mohammad Azad

Mohammad Enamul Hoque

Mohammad Eslamian

Mohammad Ghalambaz

Mohammad GhasemiGo

Mohammad Ghiasi

Mohammad Ghiasian

Mohammad H. Nadimi-Shahraki

Mohammad Imdad

Mohammad Izadi

Mohammad Javad Ebadi

Mohammad Khubeb Siddiqui

Mohammad Mahdi Rezapour Mashhadi

Mohammad Malikan

Mohammad Masjed-Jamei

Mohammad Miralinaghi

Mohammad Najafzadeh

Mohammad Reza Rezaei

Mohammad Robiul Hossan

Mohammad Sajid

Mohammad Shbool

Mohammad Shojafar

Mohammad Sohail

Mohammad Y. Abdollahzadeh Jamalabadi

Mohammad Yazdi

Mohammad Yushalify Misro

Mohammadreza Soltaninejad

Mohammed A. M. Abdullah

Mohammed A.A. Al-qaness

Mohammed Al-Smadi
Mohammed Amer

Mohammed Bahja

Mohammed Eliwa

Mohammed Hariri Bakri

Mohammed Heyouni

Mohammed Jaradat

Mohammed Jashim Uddin

Mohammed K. A. Kaabar

Mohammed M. Al-Sawalha

Mohammed M. Al-Shomrani

Mohammed Mechee

Mohammed Saad Kamel

Mohammed Salah Al-Radhi

Mohammed Salem

Mohammed Shakhatreh

Mohammed Zakarya

Mohd Azlan Shah Zaidi

Mohd Herwan Sulaiman

Mohd Salmi Md Noorani

Mohiuddin Ahmed

Mohsen Mousavi

Mohsen Pirdashti

Mohsen Saffari Pour

Mohsen Soltanifar

Mohsen Tadi

Moinak Maiti

Moises Levy

Mojtaba Ahmadieh Khanesar

Mokhtar Kirane

Molina Cabrera Daniel

Molina García Víctor

Molina-Granja Fernando

Molnar Elisabeta Ilona

Momchil Nenov

Monica Aureliana Petcu

Monica Bianchini

Monica Iordache

Mónica Rodrigues

Monika Hadaś-Dyduch

Monika Ratajczak

Monika Stoma

Montserrat Guillén

Moon Soo Kim

Moreno Concezzi

Morgan Pierre

Morikazu Nakamura

Morteza Ghorbani

Mosè Gallo

Mostafa Ghandehari

Mostafa Habibi

Mostafa Kamal Sarker 
Mostafa M. A. Khater

Mostafa Safdari Shadloo

Mostefa Mohamed-Seghir

Mourad Zribi

Mudasir Younis

Mufutau Ajani Rufai

Mugur Acu

Muhamamd E. Samei

Muhammad Aamir

Muhammad Abbas

Muhammad Ahsan Saeed

Muhammad Amer Latif

Muhammad Arif

Muhammad Arif Mahmood

Muhammad Aslam

Muhammad Aslam Noor

Muhammad Ayaz

Muhammad Azher Hassan

Muhammad Aziz

Muhammad Dauhoo

Muhammad Farman

Muhammad Fazal Ijaz

Muhammad Hamid

Muhammad Imran Tariq

Muhammad Jabir Khan

Muhammad Kamran Siddiqui

Muhammad Khan

Muhammad Nadeem

Muhammad Najib Razali

Muhammad Naveed Iqbal

Muhammad Nazam

Muhammad Omer Khan

Muhammad Qaiser Shahbaz

Muhammad Qasim

Muhammad Raja

Muhammad Riaz

Muhammad S. Abdo

Muhammad Sajjad Ali Khan

Muhammad Samraiz

Muhammad Sarwar

Muhammad Shoaid Arif

Muhammad Sulaiman

Muhammad Umair Ali

Muhammad Usman

Muhammad Wakil Shahzad

Muhammad Zaka Emad

Muhammed Mursaleen

Muhammet Deveci

Muhammet Uşak

Muhittin Yilmaz

Mumtaz Karatas
Murali Mohan Cheepu

Murat Cancan

Murat Emre Kartal

Murat Kirişci

Murat Osmanovich Mamchuev

Murugaian Senthilvelan

Mustafa Avci

Mustafa Dede

Mustafa Erguvan

Mustafa Hajij

Mustafa Inc

Mustafa Turkyilmazoglu

Mustapha Hached

Mykola Khrypchenko

Myoungju Park

Myungseob (Edward) Kim

N. C. Balaji

N. Rich Nguyen

N. Subba Reddy

Nabamita Pal

Nabil Maliki

Nabil Mlaiki

Nabil Neggaz

Nabil T. Fadai

Nader Ekramirad

Nadezda Sukhorukova

Nádia Simões

Naeem Iqbal

Naeem Saleem

Nahyun Kwon

Nai-Hua Chen

Naixue Xiong

Najaf Iqbal

Najib Kacem

Nalla Anandakumar

Nallappan Gunasekaran

Nan Song

Nand Jha

Nansong Wu

Naoki Makimoto

Napat Vajragupta

Narad Rampersad

Nasheen Nur

Nasima Begum

Natalia Manea

Natalia Nehrebecka

Natalia Nikitina

Natalia Nikolova

Natalia Rojas Perilla

Natalia Wagner

Nataliya Boyko 


\begin{tabular}{|c|c|}
\hline Nataliya Chukhrova & Nickolai Kosmatov \\
\hline Natalya Pavlovna Bondarenko & Nicola Bellomo \\
\hline Natanael Karjanto & Nicola Epicoco \\
\hline Natarajan Valliammal & Nicola Gigante \\
\hline Natasa Zivic & Nicola Tarasca \\
\hline Nathalia Tejedor-Flores & Nicolae Herisanu \\
\hline Nathalie Barroca & Nicolae Lupa \\
\hline Nathalie Sauer & Nicolae Pop \\
\hline Nathan Johnson-McDaniel & Nicolae Secelean \\
\hline Nathaniel K. Newlands & Nicolae Tudoroiu \\
\hline Navchetan Awasthi & Nicolas Dupin \\
\hline Naveed Ahmed Azam & Nicolas Langrené \\
\hline Naveen Chauhan & Nicolas Privault \\
\hline Navid Razmjooy & Nicolas Scelles \\
\hline Nawin Raj & Nicoleta Dorina Racolța-Paina \\
\hline Nazif Durmaz & Nicoleta Sirghi \\
\hline Nazim Ahmed Nazim Ahmed Choudhury & Nicoletta Fala \\
\hline Ndolane Sene & Nicolina Sciaraffa \\
\hline Nebojsa Bacanin & Nicolò Vaiana \\
\hline Necati Olgun & Nicu Bizon \\
\hline Necolae Herisanu & Nicuşor Minculete \\
\hline Nediljka Gaurina-Međimurec & Nigel D. P. Barltrop \\
\hline Neftali Nuñez & Nihal Tas \\
\hline Nehad Ali Shah & Nikhil Pal \\
\hline Neli Dimitrova & Nikita Gibanov \\
\hline Nelia P. Amado & Nikita Moiseev \\
\hline Nelson Felipe Loureiro Vieira & Nikita Osintsev \\
\hline Nelson Martins-Ferreira & Nikita Y. Lovyagin \\
\hline Nelson Tavares Da Silva & Niklas Kolbe \\
\hline Nemanja Maček & Niklas König \\
\hline Nenad Cakić & Niklas L.P. Lundström \\
\hline Nenad Filipovic & Nikola Adžaga \\
\hline Nenad Šuvak & Nikola Anđelić \\
\hline Nestor Cabrera-Munoz & Nikola Gradojevic \\
\hline Neus Garrido & Nikola Koceić-Bilan \\
\hline Neven Bulic & Nikola Lopac \\
\hline Neven Hadžić & Nikola Poljak \\
\hline Newcomb Robert & Nikola Vojnović \\
\hline Nezam Rohbani & Nikolai A. Sidorov \\
\hline Ngoc Ai Thy Nguyen & Nikolai Krivulin \\
\hline Nguyen Dinh Dung & Nikolai Kudryashov \\
\hline Nguyen Quoc Khanh Le & Nikolai Ushakov \\
\hline Nguyen The Vinh & Nikolaos Manousakis \\
\hline Nha Nguyen & Nikolaos Mitianoudis \\
\hline Nhon Nguyen-Thanh & Nikolaos Ploskas \\
\hline Nhu-Ty Nguyen & Nikolaos Polatidis \\
\hline Niaz Ghumro & Nikolaos Tsitsas \\
\hline Nica Elvira & Nikolas Tsantas \\
\hline Nicholas Fantuzzi & Nikolay A. Sidorov \\
\hline Nicholas Tsounis & Nikolay K. Vitanov \\
\hline Nicholas Vlachos & Nikolay Kazanskiy \\
\hline
\end{tabular}


Nikolay Kyurkchiev

Nikolay Manev

Nikolay Neykov

Nikolay Pavlov

Nikolina Kovačević

Nikos Bagis

Nikos Georgantzis

Nikos Pnevmatikos

Niksa Jajac

Ning Wang

Ning Zhang

Nir Sochen

Nirul Masurkar

Nizar Souayah

Noboru Niguchi

Nobuyuki Hanaki

Nodari Vakhania

Noel Perera

Noelia Bazarra

Noelia Olmedo

Noemi Schmitt

Noirrit Kiran Chandra

Noman Baloch

Nor Haniza Sarmin

Nóra Baranyai

Nora Molkenthin

Norbert Tuśnio

Norimasa Yoshida

Noson Yanofski

Nuno Bastos

Nuno Carlos Leitão

Nuno Crespo

Nuno Fortes

Nuno Miguel Fonseca Ferreira

Nuno Sousa

Nur Azam Abdullah

Nuttanan Wichitaksorn

Nwagoum Tuwa Peguy Roussel

O. S. Albahri

Oana Brandibur

Octav Olteanu

Octavian Dospinescu

Ogabi Chokri

Oh Kang Kwon

Olaf Wolkenhauer

Oláh Judit

Oldrich Sucharda

Oleg A. Markelov

Oleg Evsutin

Oleg Gutik

Oleg K. Golovnin
Oleg N. Kirillov

Oleg Rogozin

Oleg Tikhonenko

Oleg Uzhga-Rebrov

Oleg V. Zakharov

Oleh Skaskiv

Oleksii Tyshchenko

Olga Belova

Olga Kochueva

Olga Liivapuu

Olga Parshina

Olgierd Hryniewicz

Olimpia Neagu

Olivier Pironneau

Olvar Bergland

Olympia Roeva

Omar Bazighifan

Omar D. Lopez

Omar Kebiri

Omar Lakkis

Ömer Oruc

Omid Nikan

Onder Altuntas

Ondrej Krejcar

Ondrej Stopka

Onofrio Gigliotta

Onur Alp Ilhan

OPhir Nave

Orchidea Maria Lecian

Oren Efraim Nahum

Orest Artemovych

Orlov Viktor Nikolaevich

Ornella Mattei

Ornella Robutti

Osama Ahmed

Osama Alabdali

Osama Moaaz

Óscar Angulo

Oscar Danilo Montoya Giraldo

Óscar Domínguez

Oscar Valero

Osei Tweneboah

Oskar Sultanov

Oswaldo Hideo Ando Junior

Otilia Manta

Ottorino Ori

Ovidiu Cosma

Ovidiu Ivanov

Ovidiu Popescu

Owen Nicholas

Özgür Ege 
P. G. Michaelides

P. Jidesh

P. Karthikeyan

P. M.Durai Raj Vincent

P. Rajendra

P. Veeresha

Pablo Caballero

Pablo Cansado

Pablo Carbonell

Pablo Durán Santomil

Pablo Gómez-Abajo

Pablo M. Berná

Pablo Pujadas Álvarez

Pablo Sebastián Alegre Rueda

Padmanabhan Mahadevan

Palanisamy Muthukumar

Palina Prysmakova

Palle E.T. Jorgensen

Pan Shang

Panagiotis D. Michailidis

Panagiotis Dimitropoulos

Panagiotis Isigonis

Panagiotis Michailidis

Panagiotis Vassiliou

Panayiotis Tzeremes

Pankaj Agrrawal

Pankaj K. Singh

Pankaj Singh

Paola Bonacini

Paola Lamberti

Paolo Crippa

Paolo Emilio Ricci

Paolo Garza

Paolo Grigolini

Paolo Mercorelli

Paolo Musolino

Paolo Pagnottoni

Paolo Paoletti

Paolo Pin

Paolo Visconti

Paolo Zagaglia

Pao-Nan Chou

Papadimitriou Fivos

Papadopoulos Policarpos

Parikshit Dutta

Parimal Samir

Parisa Haim Faridian

Park Sangwoo

Partha P. Gopmandal

Parveen Kumar

Pasquale Candito
Pasquale De Meo

Pasquale Legato

Pasquale Marcello Falcone

Pasquale Vetro

Patricia Martinkova

Patricija Bajec

Patrick Gelß

Patrick Moylan

Patrick Sole

Patrick Trainor

Patrik Peška

Patrizia Bagnerini

Patrizio Vanella

Paul Barber

Paul Batchelor

Paul Bracken

Paul Ernest

Paul F. Slade

Paul Gagniuc

Paul Georgescu

Paul Hagelstein

Paul Juinn Bing Tan

Paul Magron

Paul Metaxatos

Paul Sotiriadis

Paul Talbot

Paul Valle

Paula Amaral

Paula M. C. Catarino

Paula Triviño-Tarradas

Paula Vasquez

Pauline Vos

Paulius Ruzgys

Paulius Skačkauskas

Paulo B. Vasconcelos

Paulo Eduardo Oliveira

Paulo Ferreira

Paulo Garrido

Paulo Goncalves

Paulo Reis Mourão

Pavel Bedrikovetsky

Pavel Ceniga

Pavel Fiala

Pavel Gapeev

Pavel Gotovtsev

Pavel Loskot

Pavel Pražák

Pavel Rajmic

Pavel Stefanovič

Pavel Trojovsky

Pavel V. Matrenin 
Pavla Dráždilová

Pavlina Jordanova

Pavol Durana

Pawaton Kaemawichanurat

Paweł Albrechtowicz

Pawel Bartoszczuk

Paweł Borkowski

Pawel Chmielinski

Paweł Cybulski

Pawel Dobrzanski

Paweł Drąg

Paweł Hanus

Paweł Jabłoński

Paweł Karczmarek

Pawel Kazibudzki

Paweł Ligęza

Paweł Lonkwic

Paweł Marcin Kozyra

Pawel Pijarski

Pawel Rotter

Paweł Sikora

Paweł Weichbroth

Pawel Zajac

Paweł Ziemba

Paweł Zmarzły

Paweł Żochowski

Pedro A. Martin Cervantes

Pedro Alonso-Jordá

Pedro Andre Prates

Pedro Espadinha-Cruz

Pedro Guilherme Rocha Dos Reis

Pedro Henrique Triguis Schimit

Pedro Macedo

Pedro Mercader

Pedro Miguel Alves Ribeiro Correia

Pedro Mucharreira

Pedro Ojeda-May

Pedro Pinho

Pedro Valero-Lara

Pejman Bahramian

Pelin Yildirim Taser

Pencho Marinov

Penelope Kalogeropoulos

Peng Guo

Peng Xu

Peng Zhao

Pengfei Jia

Penghao Wang

Peng-Yeng Yin

Pericles Panagiotou

Pertti Väisänen
Perwira Redi

Peter Arendas

Peter B. Dixon

Peter Boyvalenkov

Peter Brusov

Peter Congdon

Peter Csiba

Peter Fieger

Peter Gamnitzer

Peter Grabusts

Peter Hieber

Péter Kórus

Péter L. Erdős

Peter Lenk

Peter Malega

Peter Michael Ward

Peter Odry

Peter Organisciak

Peter Poór

Peter Stadler

Péter Tamás

Peter Van De Ven

Peter Vankúš

Peter Zweifel

Petia Koprinkova-Hristova

Petr Blaschke

Petr Coufal

Petr Domnikov

Petr Eisenmann

Petr Eliáš

Petr Kudrna

Petr Lehner

Petr Opěla

Petr Valášek

Petra Amparo López Jiménez

Petra Grošelj

Petra Murinova

Petras Rupšys

Petre Anghelescu

Petre Brezeanu

Petre Caraiani

Petre Daniel Matasaru

Petrică C. Pop

Petro Feketa

Petros Migiakis

Petya G. Nedkova

Peyman M. Moradi

Pfeifer Sanja

Philip Andrew Klobucar

Philip Broadbridge

Philip H. Kass 
Philip Kilby

Philipp J. Di Dio

Philipp Junker

Philipp Neumann

Philipp Sibbertsen

Philipp Stegmann

Philippe A. Robert

Philippe Clauss

Philippe Vieu

Phillip Yam

Phuc Bui

Piedad Tolmos Rodríguez-Piñero

Pier Gentili

Pier Nicola Sergi

Pierangelo De Pace

Pierluigi Amodio

Pierluigi Vellucci

Pierre Béjot

Pierre Patie

Pietro Boccadoro

Pietro Rigo

Pietro Sabatino

Pietro Scandura

Ping Wang

Ping-Chen Chang

Piotr Borkowski

Piotr Cyklis

Piotr Derugo

Piotr Dworniczak

Piotr Fabian

Piotr Fiszeder

Piotr Frąckiewicz

Piotr Gołębiowski

Piotr Kalita

Piotr Kulicki

Piotr Nowak

Piotr Pałka

Piotr Powroźnik

Piotr Prokopowicz

Piotr Prus

Piotr Raźniak

Piotr S. Szczepaniak

Piotr Skupin

Piotr Tauzowski

Piotr Witczak

Piotr Zwierzykowski

Piyush Kant Rai

Plácido Moreno Beltrán

Po-Hsing Tseng

Po-Keng Cheng

Poonam Kumari
Pooneh Sabouri

Pouria Behnoudfar

Pourya Pourhejazy

Pouya Agahei Pour

Po-Wei Li

Pozna Claudiu Radu

Prabal Barua

Pradeep Kumar Anand

Pradeep Kumar Bhatia

Pradeep Kumar Singh

Pradeep R. Varadwaj

Pradip Debnath

Prakash Duraisamy

Prakash Veeraraghavan

Pranav Bhounsule

Pranay Goswami

Praneel Chand

Prashant Dogra

Prashant Singh

Prasun Kumar Nayak

Pratik Joshi

Praveen Agarwal

Predrag Bejaković

Predrag Ivanis

Predrag Stanimirović

Prem Kumar Singh

Priyabrat Gochhayat

Priyank Mukesh Kumar Shah

Prosenjit Chatterjee

Przemysław Garsztka

Przemysław Koprowski

Przemyslaw Stpiczynski

Przemysław Strzelecki

Puneet Pasricha

Pushpa Rathie

Pushpendra Kumar

Puteri Suhaiza Sulaiman

Puthearath Chan

Pyke Tin

Qasem Abu Al-Haija

Qi Chen

Qi Liu

Qian Huang

Qiang Ren

Qianqian Yang

Qiao Dong

Qiaofeng Li

Qiaoping Zhang

Qibin Duan

Qichun Zhang

Qidi Peng 


\begin{tabular}{|c|c|}
\hline Qifeng Liao & Rafał Podlaski \\
\hline Qingan Qiu & Rafał Wojszczyk \\
\hline Qingguo Hong & Raffaele Carli \\
\hline Qinghe Zheng & Raffaele Chiappinelli \\
\hline Qingjie Cao & Raffaele D'Ambrosio \\
\hline Qinglin Su & Raffaele Mattera \\
\hline Qingqing Ma & Raffaele Pisano \\
\hline Qingwen Wang & Raffaele Solimene \\
\hline Qinwei Fan & Raffaella Pavani \\
\hline Qiru Wang & Rafik Absi \\
\hline Qiujiang Liu & Raghda Attia \\
\hline Qiyu Chen & Rahmat Ellahi \\
\hline Quanxin Zhu & Raid Saabni \\
\hline Quoc Bao Diep & Raimondas Ciegis \\
\hline Qurban Ali Shah Syed & Raimundas Vidunas \\
\hline Quynh Tran & Raja Balaguru \\
\hline Quy-Thinh Dao & Rajani Singh \\
\hline R. G. González Rodríguez & Rajesh Kumar Saini \\
\hline R. J. Punithgowda & Rajesh N.V. P. S. Kandala \\
\hline R. S. Jangid & Rajkumar Verma \\
\hline R. Sudhakar Pandian & Rajvikram Madurai Elavarasan \\
\hline Rabczuk Timon & Rakesh K. Parmar \\
\hline Rachel Childers & Ralf-Peter Mundani \\
\hline Rachele Anderson & Raluca Andreea Felseghi \\
\hline Radan Ivanov & Raluca Eftimie \\
\hline Radek Matušů & Ram Krishan Sharma \\
\hline Radi Romanski & Ramakant Meher \\
\hline Radomír Perzina & Ramalingam Udhayakumar \\
\hline Radoslava Kraleva & Ramandeep Behl \\
\hline Radosław Trebinski & Rameshwar Dubey \\
\hline Radoslaw Winiczenko & Rami Ahmad El-Nabulsi \\
\hline Radosław Wolniak & Ramiro S. Barbosa \\
\hline Radovan Garabík & Ramón Esteban-Romero \\
\hline Radu Constantinescu & Ramón Giraldo \\
\hline Radu Lupu & Ramon Grima \\
\hline Radu Miculescu & Ramon Gutiérrez-Sánchez \\
\hline Radu Paltanea & Ramon Vilanova \\
\hline Radu-Emil Precup & Ramona Bran \\
\hline Rafael Asorey-Cacheda & Ramūnas Česnavičius \\
\hline Rafael Bernardo Carmona Benítez & Ran Etgar \\
\hline Rafael De La Rosa Silva & Ran Li \\
\hline Rafael Lopez & Ran Zhou \\
\hline Rafael Rivera-Lopez & Randy Machemehl \\
\hline Rafael Shehu & Rangaswamy Naveen Kumar \\
\hline Rafael Socas & Ranka Stanković \\
\hline Rafael-J. Villanueva & Raoul Nigmatullin \\
\hline Rafal Drezewski & Raphael Bennett-Tennenhaus \\
\hline Rafał Kowalik & Raquel Barreira \\
\hline Rafal Madonski & Raquel Cervigón \\
\hline Rafał Michalski & Raquel Gaspar \\
\hline Rafal Nagaj & Raquel Lozano Blasco \\
\hline
\end{tabular}


Rashid Ali

Rashid Jan

Rashmi Murthy

Rasool Shah

Ratan Das

Raul Cristian Roman

Raul Curto

Raúl E. Curto

Raúl Manuel Falcón Ganfornina

Raúl Pérez-Fernández

Ravi Agarwal

Ravi Kannan

Ravi R. Mazumdar

Ravil Muhamedyev

Ravinder Kumar

Ravindra Dhuli

Ravindra Kumar

RaviShankar Sathyamurthy

Raweerote Suparatulatorn

Raymond Byrne

Raymundo Cassani

Razvan Diaconescu

Reda Dehak

Rehan Ahmed

Rehmat Ellahi

Rei Sonobe

Reinaldo Souza

Reinhard Oldenburg

Reinis Cimurs

Rekha Guchhait

Rémi Léandre

Remigijus Bubnys

Remigijus Paulavičius

Renat T. Sibatov

Renata Paksiova

Renato Bruni

Renato De Leone

Renjith V. Ravi

Reny Kunnel Chacko George

Reza Abbasi

Reza Alizadeh Kordabad

Reza Barati

Reza Hafezi

Reza Kiani Mavi

Reza Maihami

Reza Reza Mirzaie

Reza Saadati

Reza Serajian

Reza Shahbazian

Reza Taherdangkoo

Ricardo Costa Climent
Ricardo Escobar-Jiménez

Ricardo Francisco Reier Forradellas

Ricardo Granizo Arrabe

Ricardo Lopez-Ruiz

Ricardo Santos

Ricardo Vinuesa

Riccardo De Benedictis

Riccardo Mandriota

Riccardo Mel

Riccardo Minto

Richard Cebula

Richard J. Cebula

Richard J. Saykally

Richard Pinčák

Richard Pospíšil

Rija Erveš

Rimantas Kacianauskas

Rita Lima

Riyaaz Uddien Shaik

Rizwan Ali Naqvi

Robert Burduk

Robert Griffiths

Robert Kotiuga

Robert Kunst

Robert Newcomb

Robert Ojsteršek

Robert Shwartz

Robert Ślepaczuk

Robert Sousa

Robert Szasz

Robert Ulewicz

Robert Vrabel

Robert W. Zimmerman

Roberta Pellegrino

Robertas Damaševičius

Roberto A. Cammino

Roberto André Henrique De Oliveira

Roberto Bernal Jaquez

Roberto Casado-Vara

Roberto Cavoretto

Roberto Corcino

Roberto Cruz

Roberto Di Capua

Roberto Ferrara

Roberto Garra

Roberto Giambo

Roberto Rocchetta

Roberto Saia

Roberto Sanchis

Robin Hankin

Robin Haunschild 
Rodica Borcia

Rodica Ianole-Calin

Rodica Luca

Rodrigo Colnago Contreras

Rodrigo Martín San Agustín

Rodrigo Olivares

Rodrigo Salas

Rodriguez Montequin Vicente

Rodríguez-Ayerbe Pedro

Roger Käppeli

Rogério Diogne De Souza e Silva

Rogério Serôdio

Roghayeh Moallem Ganji

Rohail Hassan

Rohit Babbar

Rohit Dhariwal

Roma Kačinskaitè

Romain Dolbeau

Roman Chertovskikh

Roman Dmytryshyn

Roman Gabl

Roman Ivanovich Parovik

Roman Jaskulski

Roman Parovik

Roman Syunyaev

Roman Vavrek

Roman Wituła

Roman Yavich

Romana Rupeika-Apoga

Romána Zelkó

Romesh Saigal

Romina Fucà

Romualdas Bausys

Rong Yuan

Rongwei Guo

Ronnason Chinram

Roofia Galeshi

Roohollah Noori

Roozbeh Haghnazar Koochaksaraei

Rose Marie O. Mendoza

Rossana Mastrandrea

Rossen I. Ivanov

Rossita M. Yunus

Rostand Moutou Pitti

Rostislav Razumchik

Roy Cerqueti

Roy H. Kwon

Rozarija Mikić

Rubén Picó Vila

Rudolf Janos

Rudolf Scitovski
Rui Albuquerque

Rui Eduardo Brasileiro Paiva

Rui Feng

Rui Gao

Rui Henriques

Rui Pedro Brito

Rui Santos

Rui Silva

Rui Wang

Rui Xu

Ruifeng $\mathrm{Hu}$

Ruijin Wang

Rully Charitas Indra Prahmana

Ruman Banerjee

Ruriko Yoshida

Ruslan V. Fedorov

Russell Gerrard

Russell Thompson

Rute Almeida

Ruth Bars

Rutwig Campoamor-Stursberg

Ruzica Nikolic

Ružica R. Nikolić

Ryan Bond

Ryan D. Sullivan

Ryosuke Yoshii

Ryszard Kutner

Ryszard Pukala

Ryszard Walentyński

S. A. Belbas

S. A. Mohiuddine

S. Ali Faghidian

S. Bonnini

S. Etemad

S. Farzad Ahmadi

S. I. Bezrodnykh

S. K. Li

S. Mahdi Homayouni

S. P. Sidorov

S. Padmanabhan

S. R. Chakravarthy

S. Senthil Kumaran

S. Tauseed Saeed

Saad Fawzi Al-Azzawi

Saad Motahhir

Saba Gharehdash

Sabah Jassim

Sabina Necula

Sabina Tangaro

Sabine Wittevrongel

Sabrina Leo 
Sabrina Streipert

Sachin Kumar

Sachin Patil

Sachin Sharma

Sadibou Aidara

Sadiq Abdulhussain

Sadok Rezig

Saeed Farjami

Saeed Rafee Nekoo

Saeed Sababe

Saeid Alikhani

Saeid Jafari

Safeer Ullah

Safwan Mohd Nor

Sahaj Saxena

Saheed Olayiwola

Sahm Kim

Şahsene A. Altınkaya

Saiful Hafizah Jaaman

Saiful Rahman Mondal

Sajad Jafari

Sajid Ali

Sajid Siraj

Sakander Hayat

Saleem Abdullah

Saleh A. Bawazeer

Saleh Aiad Mohamed Omran

Saleh Mobayen

Salih Djilali

Salimon Maruf Gbadebo

Salisu Ibrahim

Sally McClean

Salman A. Khan

Salman Ahmadi-Asl

Salman Izadkhah

Salman Khalid

Salvatore Ameduri

Salvatore Esposito De Falco

Salvatore Milici

Salvatore Sessa

Sam Amiri

Sam Ganzfried

Sam Mosallaeipour

Samad Noeiaghdam

Samarjit Kar

Sambor Guze

Samet Gunay

Samet Samet Memiş

Sami Mnasri

Samir Aberkane

Samir Kumar Bandopadhyay
Samira Keivanpour

Samo Kralj

Samrand Saeidi

Samsul Ariffin Abdul Karim

Samuel Kofi Erskine

Samuel Livingstone

Samuele Antonini

Samundra Regmi

Sanatan Das

Sanaz Tabatabaee

Sanbo Qin

Sanda Micula

Sanda Renko

Sandeep Sony

Sandip Dutta

Sándor Kovács

Sándor Szénási

Sandra Ferreira

Sandra Nite

Sandra Rugonyi

Sandro Radovanović

Sandro Skansi

Sang Hyun Seo

Sang-Eon Han

Sangheon Han

Sanghyun Kim

Sangin Kim

Sangseung Lee

Sani Abba

Sanja Rukavina

Sanjay Ghosh

Sanjay R. Kharche

Sanjin Gumbarević

Sanjoy Kumar Paul

Sankar Kumar Roy

Sankar Prasad Mondal

Santanu Metia

Sante Francesco Rende

Santi Alonso-Quesada

Santiago Pozo Sánchez

Santos Omar

Santoso Wibowo

Santwana Mukhopadhyay

Sanyapong Petchrompo

Saptarshi Sengupta

Sara Grundel

Sara I. Abdelsalam

Sara Kasmaeeyazdi

Sara Nunes

Sara Ricci

Sara Shahzad 
Sarat Mohapatra

Sarbast Moslem

Sardar Khizar Hayat

Šárka Hošková-Mayerová

Šárka Hudecová

Sarka Mayerova

Sarris Ioannis

Sarunas Grigaliunas

Sascha Ranftl

Sašo Karakatič

Sathishkumar Murugesan

Satoru Morita

Satoshi Uchida

Saulius Minkevičius

Saurabh Singh

Sauvey Christophe

Saverio Maietta

Savin Treanta

Savitri Bevinakoppa

Sayantan Choudhury

Sayar Karmakar

Schweder Tore

Se Hoon Jung

Sean Holman

Sebastian Aniţa

Sebastian Berhausen

Sebastián Franchino-Viñas

Sebastian Nugroho

Sebastian Pater

Sebastian Tomczak

Sébastien Boyaval

Sebastien Jacques

Sedigheh Sabermahani

Sègla Kpodjedo

Seham Mahyoub Al-Mekhlafi

Se-Hoon Jung

Seifedine Kadry

Selene Pirola

Selim Sukhtaiev

Semën Kutateladze

Sen Qiu

Seng-Huat Ong

Senol Piskin

Senthamarai Kannan

Senthil Krishnamurthy

Seok-Zun Song

Seong Tak Woo

Seongjin Lee

Seongkwan Cho

Seo-Yoon Ryu

Serafin Lopez-Cuervo Medina
Sercan Turhan

Serdar Carbas

Serdar Durdyev

Serdar Ekinci

Serena Santis

Serge Prudhomme

Sergei Chernyi

Sergei Fedotov

Sergei I. Mukhin

Sergei Khakalo

Sergei L. Shishkin

Sergei M. Sitnik

Sergei O. Kuznetsov

Sergei S. Pilyugin

Sergei Sherbakov

Sergei Soldatenko

Sergey A. Butenkov

Sergey Barykin

Sergey Dashkovskiy

Sergey Ershkov

Sergey Gladkov

Sergey Grigor'evich Pyatkov

Sergey Ludkowski

Sergey P. Stepanov

Sergey Pethoukov

Sergey Piskarev

Sergey Shmarev

Sergey Simakov

Sergey V. Kravchenko

Sergey Victor Ludkowski

Sergey Vladimirovich Sudoplatov

Sergii Babichev

Sergii Kushch

Sergio Barile

Sergio Cacciatori

Sérgio Duarte Correia

Sergio Gomez

Sergio Luis Náñez Alonso

Sérgio Matos

Sergiy Koshkin

Serhat Yüksel

Serhii Stepenko

Serkan Araci

Seung Hwan Lee

Seung-Bae Jeon

SeungHoon LEE

Seung-Yup Jang

Sevda Yildiz

Seyed Jalaleddin Mousavirad

Seyedali Mirjalili

Seyed-Ali Sadegh-Zadeh 
Seyede Fatemeh Ghoreishi

Seyedeh Sepideh Ghaffari

Seyyed Ahmad Edalatpanah

Shaaban Abdallah

Shadi Abpeikar

Shady H. E. A. Aleem

Shahar Hod

Shaher Zyoud

Shahram Rezapour

Shahrokh Rahbari

Shahzad Ashraf

Shahzad Chaudhry

Shahzaib Ashraf

Shailesh Patel

Shakizada U. Niyazbekova

Sham'Ah Md-Yunus

Shama Islam

Shan Jiang

Shane Mueller

Shanhe Wu

Shaobo He

Shaohan Rao

Shaohui Wang

Shaomin $\mathrm{Wu}$

Shaoping Xiao

Sharfuddin Ahmed Khan

Sharief Deshmukh

Sharifu Ura

Sharon Friesen

Sharyn Livy

Shefali Saxena

Sheila García-Martín

Sheng $\mathrm{Xu}$

Sheng Zhang

Shengchao Zhuang

Shengjun Zhou

Shengkun Xie

Sheng-Wei Chi

Shengying Yue

Shen-Tsu Wang

Shervin Minaee

Sherzod Tashpulatov

Shib Sankar Sana

Shibzukhov Zaur Mukhadinovich

Shigeo Shioda

Shigeyuki Hamori

Shih-Che Lo

Shih-Hung Yang

Shijping Wan

Shijun Liao

Shinji Inoue
Shin-Li Lu

Shin-ya Matsushita

Shirley Wyver

Shiu Yin Yuen

Shixuan Wang

Shobha M. Erappa

Shomona Gracia Jacob

Shoubhik Mondal

Shouming Zhong

Shouro Dasgupta

Shouzhen Zeng

Shoya Ishimaru

Shreen El-Sapa

Shrijita Bhattacharya

Shu Wang

Shuai Liu

Shuai Zhao

Shuangming Yang

Shuangzhe Liu

Shuanhong Wang

Shuanping Dai

Shubham Gupta

Shuhuang Xiang

Shumaila Javeed

Shunli Wang

Shuo-Jye Wu

Shusen Pu

Shuvodeep De

Shuyu Sun

Shyam S. Santra

Shy-Der Lin

Siamak Hoseinzadeh

Siaw-Lynn Ng

Sihon H. Crutcher

Sijin Lin

Sikha Bagui

Silvia Carlotto

Silvia Carpitella

Silvia Dedu

Silvia Gaftandzhieva

Sílvio Gama

Silviu Urziceanu

Simeon Reich

Simm Stefan

Simon Bheki Khoza

Simon Labouesse

Simona Decu (Marinescu)

Simona Fortunati

Simona Petrakieva

Simona-Luiza Druță-Romaniuc

Simone Ferrari 
Simone Fiori

Simone Spampinati

Simone Venettacci

Sina Etemad

Sina Niazi

Sina Shokoohyar

Sinan Deniz

Singh Kulvinder

Siniša Bogdan

Sintayehu Gebre

Siraj Ul Islam

Siran Li

Siti Mistima Maat

Siu-Long Lei

SK Karthick

Sky Kiatying Seah

Slav Ivanov Cholakov

Sławomir Błasiak

Sławomir Cięszczyk

Sławomir Golak

Slawomir Gulkowski

Sławomir Milewski

Sławomir Pietrowicz

Slobodan Babic

Slobodan Đukanović

Slobodan Radojevic

Snezhana Hristova

Sobhan Moosavi

Sofia D. Anastasiadou

Sofiane Kharbech

Sofiane Kichou

Soledad Moreno-Pulido

Somasekhara Goud Sontti

Somayajulu Dhulipala

Somayeh Sharifi

Song Foh Chew

Songsong Dai

Soni M. Pradhanang

Sonia De Lucas Santos

Sonia Tarazona

Soo-Yong Kim

Sophia Jang

Sophie Plassin

Sorana Bolboaca

Sorana D. Bolboacă

Sören Möller

Sorin Burlacu

Sorin V. Sabau

Sorin Vlase

Sorinel Căpușneanu

Soroush Safarzadeh
Sotiris Kotsiantis

Souad Larabi-Marie-Sainte

Soumava Boral

Soumyajyoti Biswas

Sourav Mondal

Spandan Roy

Spiros Cotsakis

Spiros D. Dafnis

Spyridon Doukakis

Spyridon K. Chronopoulos

Spyridon Mavrakos

Srgey Simakov

Sri Adi Widodo

Srijana Acharya

Srimannarayana Grandhi

Stamatios Papadakis

Stamatis Papadakis

Stan Chirita

Stanimir Ivanov Kabaivanov

Stanislav Anatolyev

Stanislav Avsec

Stanislav Lukashchuk

Stanislav Olegovich Papkov

Stanislav Shmelev

Stanislav Yu. Lukashchuk

Stanislava Mildeova

Stanisław Bacior

Stanisław Deniziak

Stanislaw Drozdz

Stanislaw Mazur

Stanisław Noga

Stef Graillat

Stefan Balint

Ştefan Bodi

Ștefan Burciu

Ştefan Cobzas

Ştefan Cristian Gherghina

Stefan Czerwik

Stefan Groote

Stefan Gumhold

Stefan Haesen

Stefan Ivanov

Stefan Ivić

Stefan Jovčić

Štefan Kohek

Stefan Segla

Štefan Václav

Stefania Cristina Curea

Stefania Di Gangi

Stefania Espa

Stefania Tomasiello 
Stefano Biagi

Stefano Caputo

Stefano Carrino

Stefano De Marchi

Stefano Feraco

Stéfano Frizzo Stefenon

Stefano Giaccari

Stefano Marchiafava

Stefano Ricci

Stefano Serra Capizzano

Štefica Mrvelj

Stefka Bouyuklieva

Stefka Fidanova

Stelios Pappas

Stelios Psarakis

Stepan A. Tersian

Štěpán Křehlík

Stepan Tersian

Stephan Trenn

Stephane Chretien

Stéphane Clivaz

Stéphane Mussard

Stephane Puechmorel

Stephanie Clark

Stephen G. Penny

Stephen Humphries

Stephen Jordan

Stephen Lynch

Stephen Pollock

Stephen S. T. Yau

Steve Carden

Steve Fulling

Steve Miller

Steven Pudney

Steven R. Williams

Steven Schuldt

Stig Børsen Hansen

Stoil I. Ivanov

Stojan Radenovi

Stojan Radenovic

Stoyan Slavov

Stylianos Mystakidis

Şuayip Yüzbaşı

Subburaj Ramasamy

Subhadeep Mukhopadhyay

Subhash Bagui

Subhashis Hazarika

Subhi R. M. Zeebaree

Subhomoy Ghosh

Subir Ghosh

Subramanian Muthaiah
Subrata Saha

Suched Likitlersuang

Sudeep Bapat

Suha Shihab

Suiliang Huang

Sujit Kumar De

Sukono Sukono

Suleyman Tek

Suman K. Banik

Sun Young Cho

Sung Wook Paek

Sungchan Kim

Sunghae Jun

Sungho Suh

Sungwon Lee

Sungyong Choi

Sunil Kumar

Sunil Sharma

Suparerk Janjarasjitt

Suraparb Keawsawasvong

Surender Reddy Salkuti

Surender Singh

Suresh Narayanasamy

Sushil Kumar Singh

Susmit Bagchi

Suthakaran Ratnasingam

Suzan Anwar

Suzan Obaiys

Suzana Blesic

Svajone Bekesiene

Svajūnas Sajavičius

Sven Nordebo

Svetislav Savovic

Svetla Stoilova

Svetlana V. Solodusha

Svetlozar T. Rachev

Svetoslav G. Nikolov

Svetozar Margenov

Svjetlana Hess

Swamy Ponpandi

Swarnava Ghosh

Syamal Kumar Samanta

Syed Basit Ali Bukhari

Syed Furqan Qadri

Syed N.U.A. Kirmani

Sylwester Kozak

Sylwia Werbińska-Wojciechowska

Sy-Ming Guu

Szilvia Nagy

Szu-Cheng Cheng

Szymon Plewik 
T. Ganapathy

T. N. Mokaev

Tadeusz Mikolajczyk

Tadeusz Sawik

Tadeusz Szymczak

Tae-Eung Sung

Taekyun Kim

Taffese Woubishet

Taher S. Hassan

Tahir Hussain

Tahir Mahmood

Taichi Haruna

Tairan Liu

Tairidis Georgios

Taishi Muraoka

Taiwo O. Adetiloye

Takaaki Abe

Takaharu Yaguchi

Takahiro G. Yamada

Takanobu Hara

Takayuki Hibi

Takeshi Emura

Takuji Arai

Takuya Yamano

Tamar Schlick

Tamara Kisovar Ivanda

Tamara Kyrylych

Tamás Nyitrai

Tamás Orosz

Tamer Abdelmaguid

Tan N. Nguyen

Tania Pencheva

Tânia Pinto-Varela

Tanja Gologranc

Tanja Grubić Kezele

Tanmoy Chatterjee

Tansel Dokeroglu

Tanveer Ahmad Tabish

Tanweer Alam

Tao Hong

$\mathrm{Tao} \mathrm{Hu}$

Tao Yuan

Tao Zhang

Tao Zhao

Tapan Senapati

Taraprasad Bhowmick

Tarek Aboelenen

Tareq Alodat

Tareq Al-Shami

Tareq Mohammed Al-shami

Tarik Eltaeib
Tat Dat Bui

Tatiana Atanasova

Tatiana García-Segura

Tatjana Dosenovic

TBM Chowdhury

Teddy Craciunescu

Teemu Ikonen

Teerapong Suksumran

Teijiro Isokawa

Temirkhan Aleroev

Temitope Omotayo

Teodor Bulboacă

Teodor Turcanu

Teodora Catinas

Teoh Sian Hoon

Teresa A. Oliveira

Teresa Neto

Teresa Scarinci

Tero Laihonen

Tero Tuovinen

Tero Vuolio

Tetsutaro Shibata

Tetsuya Yoshinaga

Thach N. Dinh

Thái Anh Nhan

Thamo Sutharssan

Thanana Nuchkrua

Thangaraj Muthuramalingam

Thanh Nguyen

Thanh Vinh Nguyen

Thanin Sit-thiwirattham

The Anh Han

The Tien Mai

Themistokles Rassias

Theocharis Kosmas

Theodore Andronikos

Theodoros Karakasidis

Theofanis Grammenos

Theofilos Papadopoulos

Theophanes Grammenos

Thi Kim Lien Nguyen

Thiago Bazzo

Thierry Dana-Picard

Thirupathaiah Vasantam

Thomas Berrett

Thomas Eiter

Thomas Ernst

Thomas J. Lampoltshammer

Thomas K. Uchida

Thomas Kletschkowski

Thomas Li 
Thomas Michelitsch

Thomas Ott

Thomas Prellberg

Thomas Riedel

Thomas Steffen

Thomas Stemler

Thomas Toulias

Thomas Tröger

Thomas Voigtmann

Thongchai Botmart

Thurasamy Ramayah

Thyago Nepomuceno

Tiago Ribeiro

Tianju Sui

Tiantang Yu

Tianyi He

Tian-Yi Li

Tianyou Tao

Tianzhou Xu

Tibor Chovan

Tibor Dulai

Tibor K. Pogány

Tibor Szkaliczki

Tihana Škrinjarić

Tijani Apalara

Tim Brüdigam

Tim Hulsen

Timilehin Opeyemi Alakoya

Timo Siikonen

Timon Rabczuk

Timothy A. Sands

Timothy M. Young

Timothy Oladunni

Timothy Sands

Timur Karimov

Tin-Chih Toly Chen

Tin-Chun Lin

Tinggui Chen

Tirthankar Ghosal

Toan Duc Cao

Todor Stoilov

Tohid Ghanbari Ghazijahani

Tom Charnock

Tom Cuchta

Tom Lefebvre

Tom Mestdag

Tomah Sogabe

Tomas Balezentis

Tomáš Hyhlík

Tomas Lucivjansky

Tomáš Martinec
Tomas Recio

Tomáš Sadílek

Tomasa Calvo

Tomasz Dyl

Tomasz Dzido

Tomasz Gajewski

Tomasz Górecki

Tomasz Górski

Tomasz Hachaj

Tomasz Kapuscinski

Tomasz Malkus

Tomasz Niedoba

Tomasz Orczyk

Tomasz Piotr Kucner

Tomasz Poplawski

Tomasz Rak

Tomasz Rokicki

Tomasz Sowiński

Tomasz Stoeck

Tomasz Talaska

Tomasz Trzepiecinski

Tomasz Żelaziński

Tomaž Kramberger

Tomer Shushi

Tomi Koivisto

Tomislav Herceg

Tomislav Keser

Tommaso Alberti

Tommaso Lando

Tommaso Ruggeri

Tommi Sottinen

Tommy Tanu Wijaya

Tomoyuki Ichiba

Tone Lerher

Tongxing Li

Toni Toharudin

Torrecillas Jover Blas

Torsten Ullrich

Toshihiro Yamada

Toshitake Kohno

Toshiyuki Sugawa

Tounsi Abdelouahed

Toyoki Matsuyama

Traian A. Pirvu

Trevor Bihl

Trifce Sandev

Tsuyoshi Yoneda

Tuan Anh Nguyen

Tuan-Tu Huynh

Tudor Barbu

Tudor C. Ionescu 
Tugba Karabiyik

Tuhin Ghosh

Tuhin Sahai

Tunjo Perić

Tuong Trong Truong

Tushar Kanti Roy

Tyrus Berry

Tzong-Ru Tsai

Uddyalok Banerjee

Udhayakumar Ramalingam

Udo Kamps

Udo Schilcher

Udo Von Toussaint

Ugur Duran

Ugur Kadak

Ugur Tuzun

Ulderico Fugacci

Ullrich Hustadt

Ulrich Berger

Umar Muhammad Modibbo

Umberto Triacca

Umesh Chandra Gupta

Upeksha Perera

Uroš Klanšek

Úrsula Faura

Urszula Bednarz

Urszula Siedlecka

Usman Riaz

Utami Dyah Syafitri

Utkal Mehta

Utkarsh Singh

Utsav Banerjee

V. I. Klyukhin

V. María Barragán

V. Venkatesha

Václav Šmídl

Václav Uruba

Vadim Romanuke

Vagelis Plevris

Vahan Huroyan

Vahid Akram

Vahid Nasir

Vahid Parvaneh

Vaidas Lukoševičius

Vakkas Ulucay

Valderi Leithardt

Valderi Reis Quietinho Leithardt

Valentin Cardeñoso-Payo

Valentin Mateev

Valentin Osuna

Valentina Colla
Valentina Diana Rusu

Valentina E. Balas

Valentina Franceschi

Valentina Mantulenko

Valentino Santucci

Valentyn Khokhlov

Valeria Leggieri

Valeria Marraffa

Valeriano Aiello

Valerie Novitzká

Valério Antonio Pamplona Salomon

Valerio Caleffi

Valeriy Andreev

Valery Karachik

Valery Pilipchuk

Valery Sbitnev

Valery Y. Glizer

Valsyl Lozynskyi

Valter Vairinhos

Vamsi Krishna Narla

Van Linh Nguyen

Van Van Huynh

Van-Dai Ta

Vanessa Camilleri

Vanessa Robins

Vanya Ivanova

Varun Gupta

Varun Kashyap

Varvara Garneli

Vasco Ribeiro Santos

Vasile Brătian

Vasile Cirtoaje

Vasile Dogaru

Vasile Marinca

Vasile Preda

Vasile-Gabriel Iana

Vasileios Karyotis

Vasiliev Borisovich Vladimir

Vasilii Erokhin

Vasilii Zaitsev

Vasiliki Bitsouni

Vasiliki Vita

Vasilios N. Katsikis

Vasilios Plakandaras

Vasilis Bellos

Vasilis Dimitriou

Vasilis P. Koutras

Vasiliy Elagin

Vasiliy Kachalov

Vasily E. Tarasov

Vassil Sgurev 
Vassilis C. Gerogiannis

Vassilis Makrakis

Vassily Lyubetsky

Vasyl Lytvyn

Vasyl Martsenyuk

Vedat Saut Erturk

Vedran Slapničar

Vedrana Jerković Štil

Velika Dragieva

Velin Andonov

Velislava Lyubenova

Velusamy Kavitha

Velusamy Vijayakumar

Venkatasamy Sureshkumar

Venkatesan Rajinikanth

Vera Gelashvili

Veronica Demenescu

Véronique Cariou

Vesa Kuikka

Veselina Bureva

Vesselin Drensky

Viacheslav Alexandrovich Artamonov

Viacheslav Artamonov

Vicenc Font Moll

Vicente Jara Vera

Vicente Martínez García

Vicente Munoz

Victor A. Skormin

Víctor Alfaro

Víctor Amor-Esteban

Victor Buttigieg

Victor Eremeyev

Victor G. Alfaro-Garcia

Victor Goman

Victor Grigoras

Victor H. De La Pena

Victor H. Moll

Victor Kagalovsky

Victor Pereira

Victor Rukavishnikov

Victoria Kurushina

Victoria López

Victoria Vysotska

Vida Davidavičienè

Vidas Raudonis

Viera Papcunová

Viet-Thanh Pham

Vijay Kumar Kukreja

Vijay Victor

Viktor Blåsjö

Viktor Freiman
Viktor Reshniak

Viktor Trynchuk

Viktor Valentinovich Vlasov

Viktor Vegh

Viliam Durišs

Viliam Kováč

Vinay Singh

Vincent Vajnovszki

Vincenzo Aquilanti

Vincenzo Ciancio

Vincenzo Deufemia

Vinícius Francisco Wasques

Vinodh Chellamuthu

Violeta Keršulienè

Viorel Minzu

Viorel Paleu

Viorica Chirila

Virendra Singh Chouhan

Virgil Dobrota

Vishnu Saseendran

Vita Jagrič

Vitali Liauchuk

Vitaliy L. Kamynin

Vitaliy Yakovyna

Vitaly Levashenko

Vitaly Volpert

Vito Bobek

Vitor Ambrósio

Vittoria Bruni

Vittoria Sposini

Vittorio Romano

Vlad Barbu

Vlad Diaconita

Vlad Muresan

Vlad Stefan Barbu

Vladimir A. Grishagin

Vladimir Al. Osipov

Vladimir Anisimov

Vladimir B. Zelentsov

Vladimir Dobrushkin

Vladimir E. Fedorov

Vladimir I. Semenov

Vladimir Jotsov

Vladimir Kodkin

Vladimir Kodnyanko

Vladimir Lipp

Vladimir Maksimov

Vladimir Mazalov

Vladimir N. Potapov

Vladimir O. Soloviev

Vladimir Răsvan 
Vladimir Rykov

Vladimir Sreckovic

Vladimir Tadić

Vladimir V. Ulyanov

Vladimir Vasilyev

Vladimir Vishnevsky

Vladimir Zelevinsky

Vladimiras Dolgopolovas

Vladislav Chubinskiy-Nadezhdin

Vladislav Nezevak

Vladislav Skorpil

Vlase Sorin

Volker Grimm

Volodymyr Mosorov

Volodymyr Ponomaryov

Volodymyr Puzyrov

Voon-Loong Wong

Vsevolod Bohaienko

Vsevolod Vladimirov

Vugar Ismailov

Vyacheslav R. Misko

Vytautas Bučinskas

Wadim Stielkowski

Wadim Strielkowski

Wael Mohammed

Waheed Ur Rehman

Wahyu Caesarendra

Wai-Lun Lo

Wajeeh Daher

Wajih Ezzeddine

Walaa Khalaf

Waleed Abdelhamed

Waleed M. Abd-Elhameed

Waleed Mugahed Al-Rahmi

Wali Khan

Walter Castro

Wan-Liang Wang

Wanquan Liu

Warren Vaz

Warrick R. Dawes

Wasim Jamshed

Wasiur R. KhudaBukhsh

Watcharaporn Cholamjiak

Wathek Chammam

Wayne Hayes

Wei Cao

Wei Gu

Wei Ning

Wei Wang

Wei Xing

Wei-Chiang Hong
Weiguo Fang

Weiguo Zhang

Wei-Lun Tsai

Weimin Han

Wei-Nan Zhang

Weiping Fu

Weiping Li

Weiqi Ji

Wei-Shih Du

Wei-Yu Chiu

Weizhong Tian

Wen Chen

Wenan Yuan

Wenchi Shou

Wendy Cho

Weng Hoe Lam

Weng Marc Lim

Weng Siew Lam

Wengui Yang

Wenhao Gui

Wenjun Jiang

Wenjun Liu

Wenkai Li

Wennan Zou

Wenning Wang

Wenqing Wang

Wen-Ran Zhang

Wentao Ma

Wen-Xiu Ma

Wenying Yao

Wenzhuo Cao

Werner Benger

Weronika Kruszelnicka

Wicak Ananduta

Wieslaw A. Dudek

Wiesław Alejziak

Wiesław Leoński

Wiktor Malesza

Willem Van Driel

William Martens

William McKiver

William Steingartner

William Sulis

William Yu Wang

William Zahner

Wilma Polini

Wilson Pavón

Wim Van Den Noortgate

Wing-Yee Lo

Winston Sweatman

Witold Cecot 
Witold Chmielarz

Witold Kosmala

Witold Orzeszko

Włodzimierz Fechner

Wojciech Chachólski

Wojciech Kempa

Wojciech Kryszewski

Wojciech Sałabun

Wojciech Sumelka

Won Kwang Park

Wong Foek Tjong

Woo Kai Yin

Wookjae Heo

Woong Lim

Wooram Kim

Woosuk Shin

Wudhichai Assawinchaichote

Wujun Si

Wuming Liu

Xabier Domínguez

Xabier Insausti

Xavi Barber

Xiang Gao

Xiang Li

Xiang Ma

Xiang Peng

Xiang Zhang

Xiangcheng Zheng

Xiangmin Jiao

Xiangsheng Gao

Xiangyang $\mathrm{Xu}$

Xianming $\mathrm{Gu}$

Xian-Ming Zhang

Xianyang Meng

Xiao Chen

Xiao Li

Xiaobai Meng

Xiaofeng Li

Xiaojun (Gene) Shan

Xiao-Kang Wang

Xiao-Lei Shi

Xiaolin Wang

Xiaolong Qin

Xiaoqiao He

Xiaoqing Eleanor $\mathrm{Xu}$

Xiaosheng $\mathrm{Yu}$

Xiaowu Li

Xiaoyi Zhang

Xiaoying Zhuang

$\mathrm{Xin} \mathrm{Yu}$

Xindong Peng
Xinge You

Xingwei LI

Xingwu Chen

Xingxing $\mathrm{Zu}$

Xingyu Zhao

Xinqiang Chen

Xinran Ruan

Xintian Liu

Xinwei Yu

Xu-dong Zhao

Xuefeng Zhang

Xueliang Wang

Xue-Ming Yuan

Xuewei Zhang

Xun Li

Xuyang Zhang

Y. H. Qian

Y. T. Gu

Yafei Wang

Yagub Sharifov

Yajuan Yu

Yajun Guo

Yan Lu

Yan Sun

Ya-Nan Zhou

Yanfeng Zhao

Yang (Felix) Lou

Yang Kan

Yang Pan

Yang Shen

Yang Song

Yang Yu

Yang Zhang

Yang-Quan Chen

Yangxin $\mathrm{Yu}$

Yanlin Shi

Yann Danlée

Yannis Haralambous

Yanpeng Zhang

Yanping Chen

Yanqiao Wei

Yansheng Liu

Yan-Tsung Peng

Yao Zhang

Yaodong Gu

Yaozhong $\mathrm{Hu}$

Yaqoob Majeed

Yaroslav Sergeyev

Yaroslavna Pankratova

Yaser Kiani

Yashar Tarverdi 


\begin{tabular}{|c|c|}
\hline Yasin Wahid Rabby & Yoshihiro Hamaguchi \\
\hline Yasir Khan & Yoshihiro Sawano \\
\hline Yasunari Matsuzaka & Yoshinori Shiihara \\
\hline Yeliz Karaca & Yoshiyuki Yabuuchi \\
\hline Yenming J. Chen & Youliang Tian \\
\hline Yen-wei Chen & Young Bae Jun \\
\hline Yeol Je Cho & Young Ho Kim \\
\hline Yeong-Nan Yeh & Young Jae Sim \\
\hline Yessica Nataliani & Young Suh Kim \\
\hline Yeyin $\mathrm{Xu}$ & Youngchul Bae \\
\hline Ye-Young Moon & Youri Kabanov \\
\hline Yi Fang & Youseef Alotaibi \\
\hline Yi Liang & Yousef (Joseph) Ghaffari Motlagh \\
\hline Yi Zhang & Youssri Youssri \\
\hline Yi Zhao & Yu Zhang \\
\hline Yi Zuo & Yu Zhong \\
\hline Yifan Lu & Yuanhua Ni \\
\hline Yifei Guan & Yuankai Li \\
\hline Yi-Hsien Wang & Yuan-Wei Tseng \\
\hline Yijun Lou & Yuanxin Li \\
\hline Yi-Kuei Lin & Yu-chen Liu \\
\hline Yilmaz Simsek & Yucheng Fu \\
\hline Yilun Shang & Yu-Cheng Wang \\
\hline Yiming Chen & Yuchu Qin \\
\hline Yingjie Liang & Yu-da Lin \\
\hline Yingtao Liu & Yudong Zhang \\
\hline Yingying $\mathrm{Xu}$ & Yuegang Tao \\
\hline Yiqun Xie & Yuen Kum Fai \\
\hline Yiu Yin Raymond Lee & Yuewu Wang \\
\hline Yixing Li & Yufei Zhang \\
\hline Yixuan Feng & Yuhang Zhou \\
\hline Yiyang Chen & Yuhlong Lio \\
\hline Yiying Zhang & Yu-Hsi Huang \\
\hline Yllka Velaj & Yu-Hsi Yuan \\
\hline Yoh Iwasa & Yuichi Hirose \\
\hline Yolanda Larriba & Yu-Jing Chiu \\
\hline Yong Deng & Yuki Toyoshima \\
\hline Yong Peng & Yulia Vertakova \\
\hline Yongdae Kim & Yulian Cao \\
\hline Yong-Ge Yang & Yulong Sun \\
\hline Yonghee Kim & Yun Liu \\
\hline Yonghong Yao & Yun Shang \\
\hline Yonghua Yan & Yun Yang \\
\hline Yongkun Li & Yun Yuan \\
\hline Yong-Li Sun & Yuncai Yu \\
\hline Yongshun Liang & Yunchen Yang \\
\hline Yongzhu Xiong & Yung Hsiang Chen \\
\hline Yorgos Marinakis & Yung Po Tsang \\
\hline Yoritaka Iwata & Yun-Ho Kim \\
\hline Yoshifumi Manabe & Yunming Wang \\
\hline Yoshifumi Muroi & Yunsik Kwak \\
\hline
\end{tabular}




\begin{tabular}{|c|c|}
\hline Yunus Egi & Zebin Zhao \\
\hline Yuping Gao & Zeda Li \\
\hline Yuping Li & Zeinab Shahbazi \\
\hline Yuri Dimitrov & Zélia Da Rocha \\
\hline Yuri Gliklikh & Željka Milin Šipuš \\
\hline Yuri Kondratiev & Željko Stević \\
\hline Yuri Luchko & Zenqiang Chen \\
\hline Yuri S. Popkov & Zeyad Al-Shibaany \\
\hline Yuri Tyutyunov & Zhaopeng Hao \\
\hline Yurii Averboukh & Zhekang Dong \\
\hline Yurii Lovyagin & Zhelyo Zhelev \\
\hline Yurij Povstenko & Zheming Wang \\
\hline Yuriy Bilan & Zhen $\mathrm{Wu}$ \\
\hline Yuriy Povstenko & Zhen Zhang \\
\hline Yuriy Shablya & Zhengjiang Zhang \\
\hline Yuriy Syerov & Zhengjun Liu \\
\hline Yury K. Mashunin & Zhengkui Wang \\
\hline Yury S. Khokhlov & Zhengwei Sun \\
\hline Yury Shestopalov & Zhensheng Wang \\
\hline Yusuf F. Zakariya & Zhenyu Zhao \\
\hline Yusuf Yesilce & Zhi He \\
\hline Yuta Hanazawa & Zhibin Wu \\
\hline Yutaka Okabe & Zhicheng Qiu \\
\hline Yuto Miyatake & Zhifu Tao \\
\hline Yuvaraja Teekaraman & Zhihai Rong \\
\hline Yuvraj Gajpal & Zhihua Wang \\
\hline Yuwei Wang & Zhihuo Xu \\
\hline Yuxiang Sun & Zhiming Zhang \\
\hline Yuxing Li & Zhiqian Chen \\
\hline Yuzhu Guo & Zhiqiang Huo \\
\hline Yuzuru Kato & Zhi-Qin Xu \\
\hline Zacharoula Kalogiratou & Zhiqun Xie \\
\hline Zahir Hussain & Zhiyong Sun \\
\hline Zahir M. Hussain & Zhiyuan Li \\
\hline Zahir Shah & Zhong Guozhang \\
\hline Zahra Foroozandeh & Zhongbao Wei \\
\hline Zahra Pooranian & Zhongxiang Chen \\
\hline Zailan Siri & Zhongyun Hua \\
\hline Zakaria Meliani & Zhuojia Fu \\
\hline Zakia Hammouch & Zhuoqin Yang \\
\hline Zaoli Yang & Ziad Saghir \\
\hline Zbăganu Gheorghiță & Žiga Gosar \\
\hline Zbigniew Leonowicz & Zihao Zhang \\
\hline Zbigniew Lesniak & Ziheng Shangguan \\
\hline Zbigniew Nadolny & Zijing Wong \\
\hline Zbigniew Pozorski & Zi-Ke Zhang \\
\hline Zbysław Dobrowolski & Zishun Liu \\
\hline Zdeněk Kala & Ziwei Ma \\
\hline Zdenka Zakova Kroupova & Zixiang Li \\
\hline Zdzisław Dzedzej & Zlatin Zlatev \\
\hline Ze Jin & Zohair Al-Ameen \\
\hline
\end{tabular}


Zohreh Sheikh Khozani

Zoltán Gal

Zoltan German-Sallo

Zoltán Léka

Zoltán Szilasi

Zsolt Gulácsi
Zsolt Tibor Kosztyan

Zulqurnain Sabir

Zuohui Fu

Zuzana Patikova

Zvi Lerman

Zvi Wiener 